\title{
Death assemblages of the freshwater mussels Unio crassus and $U$. tumidus (Bivalvia, Unionidae) from southern Finland: comparing taphonomical data with ${ }^{14} \mathrm{C}$ dates
}

\author{
Jan Kresten Nielsen \& Samuli Helama
}

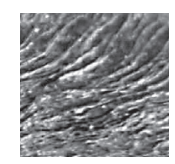

\begin{abstract}
Populations of the freshwater mussels Unio crassus and U. tumidus have declined significantly over many decades. The river Vantaanjoki, at their northern limit in southern Finland, has yielded a number of empty hinged shells of U. crassus ( 39 shells) and $U$. tumidus ( 71 shells), which have been studied visually for taphonomical features. All of the shells are to some degree affected by taphonomical processes, particularly dissolution and fragmentation. These death assemblages of Unio shells are time-averaged as suggested by their radiocarbon $\left({ }^{14} \mathrm{C}\right)$ dates, with consequences for the expected temporal resolution of both death and fossil assemblages. Although the shells are of different ontogenetic and post-mortem ages, there are no distinct differences in their preservation. Considering both taphonomical features and ${ }^{14} \mathrm{C}$ dating, time-averaged death assemblages, which might illustrate the time-evolving anthropogenic changes of the river environment over the last centuries, should with some caution be compared with modern populations for conservation evaluations. - Key words: Bivalvia, death assemblages, taphonomy, radiocarbon dates, time-averaging.
\end{abstract}

Nielsen, J.K. \& Helama, S. 2021. Death assemblages of the freshwater mussels Unio crassus and U. tumidus (Bivalvia, Unionidae) from southern Finland: comparing taphonomical data with ${ }^{14} \mathrm{C}$ dates. Bulletin of Geosciences $96(4)$, 459-480 (4 figures, 8 tables). Czech Geological Survey, Prague. ISSN 1214-1119. Czech Geological Survey, Prague. ISSN 1214-1119. Manuscript received May 6, 2020; accepted in revised form May 13, 2021; published online July 18, 2021; issued October 1, 2021.

Jan Kresten Nielsen, Süleyman Demirel University, Department of Geological Engineering, 32260 Isparta, Turkey; taphofacies@hotmail.com • Samuli Helama, Natural Resources Institute Finland, Ounasjoentie 6, 96200 Rovaniemi, Finland

The freshwater mussels Unio crassus Philipsson, 1788 and U. tumidus Philipsson, 1788 (family Unionidae Rafinesque 1820) live buried in the river bottom of coarse-grained sediment (e.g. Wesenberg-Lund 1937). Historical notes show that, the mid- $19^{\text {th }}$ century, $U$. crassus was widely spread across Europe (e.g. Nordenskiöld \& Nylander 1856, Westerlund 1871-1873), these observations including the population recorded in several sites in southern Finland (Nordenskiöld \& Nylander 1856), but more recently the species $U$. crassus is classified as Endangered on the IUCN Red List of Threatened Species (Lopes-Lima et al. 2014, 2017) and Vulnerable on the Red List of Finnish species (Hyvärinen et al. 2019). Another Unio species, $U$. tumidus is included in the category Least Concern on the IUCN European Red List of non-marine molluscs (Cuttelod et al. 2011, Van Damme 2011). However, also this species is rapidly declining in western part of its range (Van Damme 2011, Lopes-Lima et al. 2017) and, as comprehensively summarised by Lopes-Lima et al. (2017), U. tumidus is classified as Endangered at national level in Germany and Switzerland, Vulnerable in Austria, Czech Republic, Romania and Slovakia, and Near Threatened in Great Britain.

The decline in the recent populations limits the possible retrievement of living river mussels for research purposes. By contrast, death and fossil assemblages of shells representing endangered species can be located and provide shells of palaeontological origin from the populations that still exist. There are several reasons for applying palaeontological techniques to such shell collections. Foremost, studying specimens of dead individuals is an ethical and appropriate way to increase our understanding on an endangered species, such as $U$. crassus and, albeit to a lesser degree, U. tumidus. Death and fossil assemblages are also useful for understanding the former distribution of freshwater mussels such as $U$. crassus and $U$. tumidus in areas there they now are extinct (e.g. Nielsen et al. 2008). Studying dead individuals is also essential for understanding the potential ways the shells from death and fossil assemblages may 


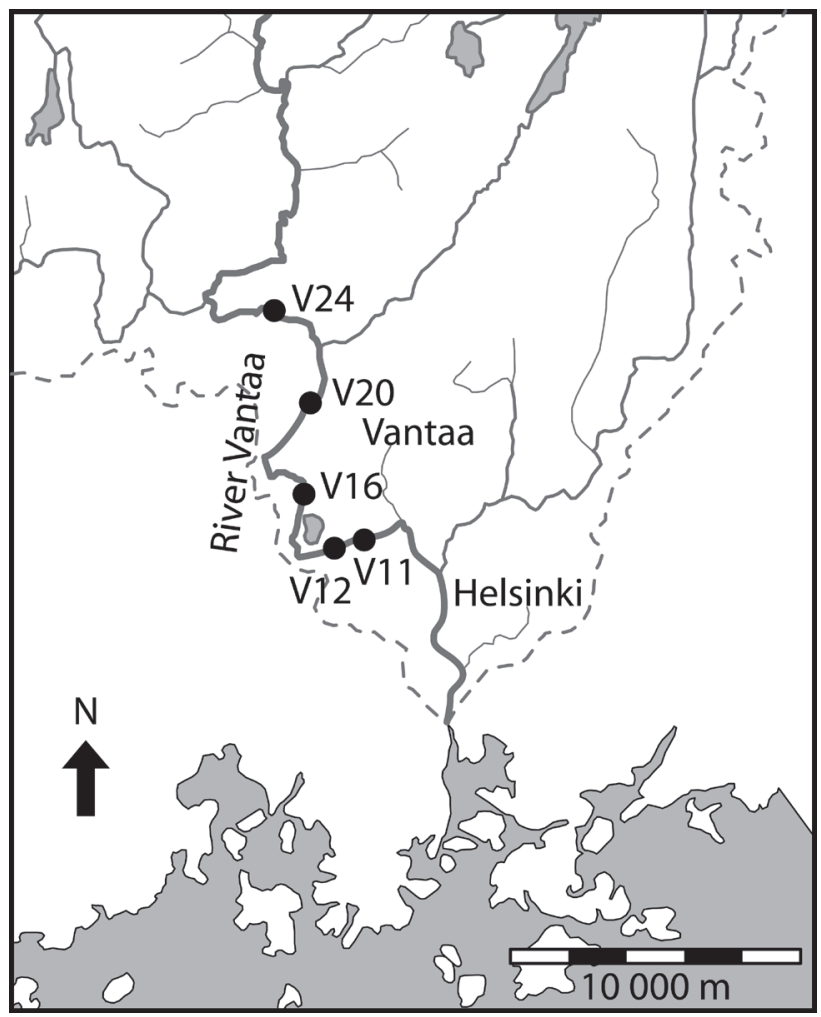

Figure 1. Geographical map with investigated locations of Unio crassus and $U$. tumidus in the River Vantaa, southern Finland. Modified from Vahtera et al. (2010).

differ from those collected from living assemblages, thus not simply assuming the condition of the dead shells to reflect those from living assemblages, as the taphonomical processes may have markedly altered the physical appearance of fossil specimens. The hard parts including calcareous shell material, ligament and periostracum can be related to aspects of autoecology, ecological systems and climate changes in the past (e.g. Fürsich \& Kauffman 1984, Helama \& Valovirta 2014). Moreover, findings of the death assemblages depend upon the differential preservation of the hard parts (e.g. Driscoll 1970, Nielsen et al. 2008, Wolverton et al. 2010). Also, a better understanding of shell deterioration before final burial may be crucial for locating additional death and fossil assemblages. The aim of this study is therefore to evaluate the preservation potential of death assemblages of $U$. crassus and $U$. tumidus from the River Vantaa in southern Finland (Fig. 1), and thus contributing to the foundation for conservation palaeobiology (e.g. Flessa 2002; Willis \& Birks 2006; Kidwell 2013, 2015; Dietl et al. 2015; Kusnerik et al. 2020), as recently pursued for shells of different freshwater mussel species in Finland (Helama et al. 2007, 2017). According to Dietl et al. (2015), conservation palaeobiology refers to a discipline that uses geohistorical data to test the hypotheses and models of how biota responds to environmental stressors, to meet the challenges of sustaining and restoring the ecosystem services. Previously, the $U$. crassus shells from this river were analysed for their growth characteristics by means of shell growth increment data (Helama et al. 2017). Thus far, there are no taphonomical studies of $U$. crassus or $U$. tumidus shells, however, for these sites, and we are not aware of any studies concentrating on their taphonomy elsewhere, despite the high conservative status and concomitantly obvious needs to analyse their conchological characteristics to derive implications for conservation and to maximise the impact of conservation measures.

\section{Study area}

The mainstream of River Vantaa is $99 \mathrm{~km}$ long, and flows $111 \mathrm{~m}$ lower into the Gulf of Finland at the bay of Vanhankaupunginselkä, in Helsinki (Tikkanen 1989). The catchment area is about $1680 \mathrm{~km}^{2}$ in the regions of Uusimaa and Etelä-Häme, belonging to Finland's most densely populated area (Tikkanen 1989, Vahtera et al. 2010). Soils of the basin of the River Vantaa comprise silt and clay (39\%), till (25\%), gravel and sand (20\%), peat $(9.4 \%)$ and bedrocks $(7 \%)$. The bedrocks and derived sediments are pyroxene gneiss, granite and granodiorite, and vulcanite, tuffite and amphibolite.

According to field surveys by Pekkarinen $(1991,1993)$ and Valovirta (2008), there are six species of freshwater bivalves inhabiting the river today: U. crassus, U. tumidus, U. pictorium, Anodonta cygnea, A. anatina and Pseudanodonta complanata. Until the early $20^{\text {th }}$ century, Margaritifera margaritifera also occurred in the same river (Valovirta 2008). Our shells of $U$. crassus originate from the sites V11 through V20 in River Vantaa (see Fig. 1), whereas all the shells of $U$. tumidus represent the site V12. Monitoring of water quality was done in the period 2006-2009 (Vahtera et al. 2010). These data are available from the sites V16 and V24, in the Vantaa municipality. The site V16 is on the same river stretch as the other of our shell sites whereas the site V24 is located farther upstream. As reference to the available environmental observations at or near the sites, for the years 2006-2009, annual averages of periodic water measurements carried out at the site V16 show temperature $11{ }^{\circ} \mathrm{C}$, oxygen $10-11 \mathrm{mg} / 1, \mathrm{pH} 7.4-7.7$, conductivity $17-23 \mathrm{mS} / \mathrm{m}$, opacity 27-67 FTU. At the site V24, the averages are comparable, namely, temperature $9.7-11{ }^{\circ} \mathrm{C}$, oxygen $10 \mathrm{mg} / \mathrm{l}$, $\mathrm{pH} 7.3-7.5$, conductivity $17-22 \mathrm{mS} / \mathrm{m}$, opacity $26-54 \mathrm{FTU}$.

\section{Material and methods}

Empty hinged shells of $U$. crassus and $U$. tumidus were previously collected (hand-picked) by divers from death 

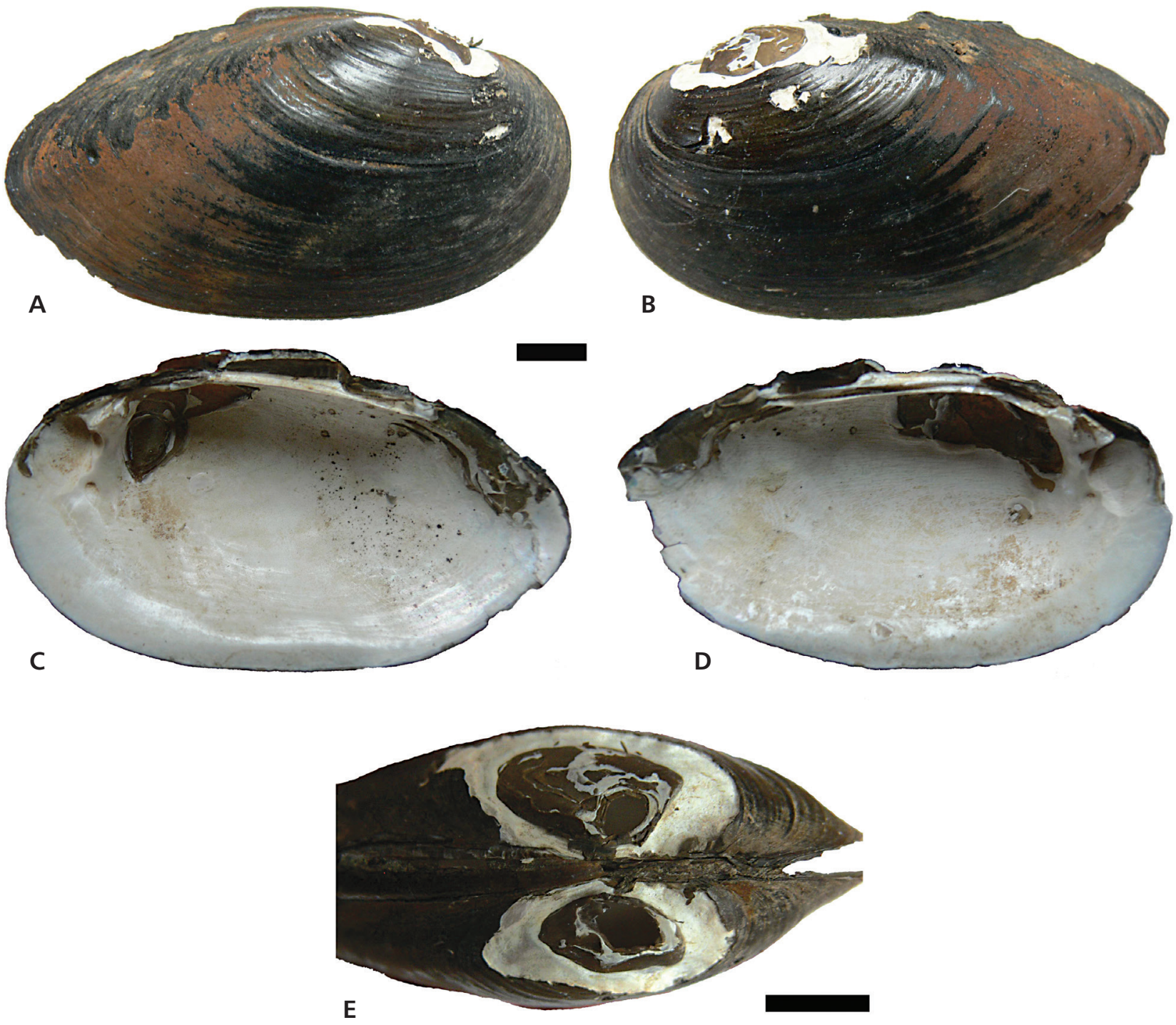

Figure 2. Unio crassus (specimen UC17-002). A, B - outer shell surface of right and left valves. The periostracum is well preserved, expect in the umbonal areas. C, D - inner shell surface of right and left valves. The inner surface is partly dull, i.e. slightly chalky, and partly gentle lustre in places. $\mathrm{E}$ - dorsal view of the left and right valves. The ligament is well preserved, while the umbonal areas are severely dissolved. Scale bars $10 \mathrm{~mm}$.

assemblages from the river sediment during the fieldwork campaigns, between 2004 and 2007, and added to the collections of the Finnish Museum of Natural History (University of Helsinki) (Valovirta 2008) (Figs 2, 3). The fieldwork, which was done in co-operation with WWFFinland and under the licences to officially collect the shell material, has been previously described in more details in Valovirta (2008) and Helama et al. (2017). As a premise, the shells were not hypothesised being deposited in the sediment for decades, probably less than a decade, and they were consequently assumed to represent modern radiocarbon ages (i.e. post-1950 cal. AD). To test the hypothesis, the shell material from the ventral margin of seven valves of $U$. crassus were radiocarbon $\left({ }^{14} \mathrm{C}\right)$ dated by the Poznań Radiocarbon Laboratory (Goslar 2010). The program OxCal version 4.3.2 (Bronk Ramsey 2009) was applied for calibration. This process can also involve the ${ }^{14} \mathrm{C}$ bomb signal, referring to a spike in the quantity of radiocarbon in the earth's atmosphere, and hydrosphere, as a result from thermonuclear bomb testing era, and leaving a detectible signature in calcified structures of organisms living in the 1950s and 1960s (e.g. DavisFoust et al. 2009). In the case of pre-bomb ${ }^{14} \mathrm{C}$ samples, the calibration was done using IntCal13 for the northern hemisphere (Reimer et al. 2013), while the post-bomb ${ }^{14} \mathrm{C}$ samples were calibrated to the curve Bomb13 Northern Hemispheric zone 1 (NH1) for north of latitude $40^{\circ} \mathrm{N}$ (Hua et al. 2013). From the bomb-curve calibration, the 
values of percent measured carbon (pMC) were expressed as the fraction modern carbon $\left(\mathrm{F}^{14} \mathrm{C}\right.$, Reimer et al. 2004), that is, $\mathrm{F}^{14} \mathrm{C}=\mathrm{pMC} / 100$ (e.g. Stenström et al. 2011, Larsen et al. 2018).

The sample sets of Unio crassus and U. tumidus are constituted by 39 and 71 shells, respectively. All the valves of $U$. crassus and $U$. tumidus have been collected as pairs, that is, hinged valves. The dimensions of anterior-posterior shell length, dorso-ventral shell height and the extent of umbonal dissolution were measured with a digital calliper with $0.01 \mathrm{~mm}$ resolution and $0.02 \mathrm{~mm}$ accuracy. Area affected by dissolution was measured along the anteriorposterior length of the valves as well as parallel to the dorso-ventral height. These measurements followed the previously established ways to document the freshwater shell dimensions of the same species (Björk 1962, Helama \& Valovirta 2007, Helama et al. 2017). Taphonomical features of individual valves were studied under binocular microscope and recorded semi-qualitatively after a threelevel scheme of taphonomical grades (e.g. Kowalewski et al. 1995, Hallman et al. 1996, Nielsen \& Funder 2003). This was done for the fragmentation, dissolution of the outer shell surface and umbonal areas, dissolution and lustre of the inner shell surface, periostracum in (non-) umbonal areas, and ligament. Here, lustre refers to the shell microstructure with refractive properties observed on the surface of the nacre, creating the iridescent lustre (Szabó 2017), that is gradually lost through the postmortem processes. Absent, moderate and high alterations were scored as the grades 0,1 and 2, respectively. With these scores, the grade 2 refers to alteration of greater than $20 \%$ valve surface, except for fragmentation, where the boundary between grades 1 and 2 is at $50 \%$. Because of the relatively well-preserved periostracum, other features on the outer shell surface of the non-umbonal areas were generally kept unobserved in order not to damage the investigated material. Grades are plotted in ternary taphograms, that is, triangular diagrams, to illustrate their variation at each site (Kowalewski et al. 1995, Hallman et al. 1996).

Subsequently, the data were evaluated statistically. The taphonomical grades were defined differently for fragmentation so that the grades may be non-linearly related between the taphonomical features. Spearman's $r$, was used to estimate the relatedness of the grades. These calculations were carried out by using the software PAST (Hammer et al. 2001, Hammer 2018), in which for computation of the calculated coefficients refers to Press et al. (1992). To estimate the significance of the Spearman's coefficients, we follow Ramsey (1989) who estimated critical values based on the Edgeworth approximation. Reading from Ramsey's (1989) table, their critical values for 0.95 quantile are about 0.267 and 0.198 for $U$. crassus and $U$. tumidus, respectively. Taphonomical indices have shown to be useful in integrating a range of data available on the taphonomical features in dead-collected shells (e.g. Wesselingh et al. 2006, Gomez et al. 2009). The taphonomical indices, coined here as taphoSUM, perioSUM, and dissUMBO, were calculated based on the results representing the taphonomical features as shown in Tables 1 and 2. The taphoSUM, perioSUM, and dissUMBO indices were calculated as follows:

$$
\begin{gathered}
\text { taphoSUM }=\mathrm{F}+\mathrm{D}+\mathrm{LU}+\mathrm{P}_{\mathrm{u}}+\mathrm{P}_{\mathrm{nu}}+\mathrm{LI} \\
\text { perioSUM }=\mathrm{P}_{\mathrm{u}}+\mathrm{P}_{\mathrm{nu}}+\mathrm{LI} \\
\operatorname{dissUMBO}=\left(\mathrm{L}_{\mathrm{u}} / 2\right) \times\left(\mathrm{H}_{\mathrm{u}} / 2\right) \times \pi
\end{gathered}
$$

where $F$ is the grade of fragmentation, $D$ is the grade of dissolution of the inner surface, $L U$ is the grade of lustre of the inner surface, $P_{u}$ is the grade for umbonal periostracum, $P_{n u}$ is the grade for non-umbonal periostracum, $L I$ is the grade for the ligament, $L_{u}$ is the length of the umbonal dissolution area, and $H_{u}$ is the height of the umbonal dissolution area.

\section{Results}

\section{Shell dimensions}

The valves of Unio crassus range from 33.7 to $82.9 \mathrm{~mm}$ in anterior-posterior length $(\mathrm{N}=22$, mean $58.9 \mathrm{~mm}$, std. dev. 15.6, skewness -0.01 ) and from 18.7 to $43.8 \mathrm{~mm}$ in dorso-ventral height (left valves $\mathrm{N} 38$, mean $32.3 \mathrm{~mm}$, std. dev. 6.4, skewness -0.31) (Tabs 1, 2, 3). Linear regression with least squares was used to model this relationship statistically and was found to show a strong positive relationship (not shown).

The Unio tumidus valves are from 40.3 to $77.8 \mathrm{~mm}$ in anterior-posterior length $(\mathrm{N}=51$, mean $60.2 \mathrm{~mm}$, std. dev. 8.5, skewness -0.06 ), while they exhibit a range of values from 21.4 to $38.3 \mathrm{~mm}$ for dorso-ventral height (N 71, mean $29.6 \mathrm{~mm}$, std. dev. 3.5, skewness 0.02) (Tabs $1,2,3)$. A strong positive linear relationship was also found between these dimensions.

\section{Taphonomical features}

The shells of Unio crassus and U. tumidus were evaluated for their taphonomical features (Tabs 1, 2; Fig. 4). Evidence of abrasion (mechanical process capable of damaging the shells by abrasive agents such as river load), bioerosion (erosion by biogenic agents) and encrustation (biogenic coating on shell surface) is absent, except for a single case of caddis larvae on the dorso-posterior 

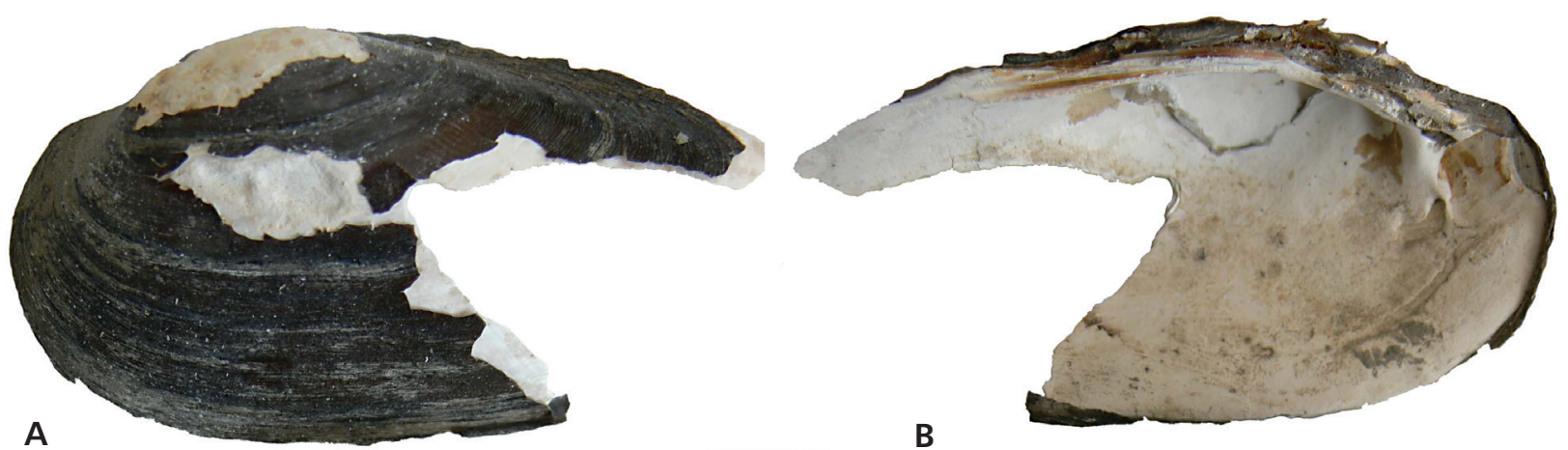

A
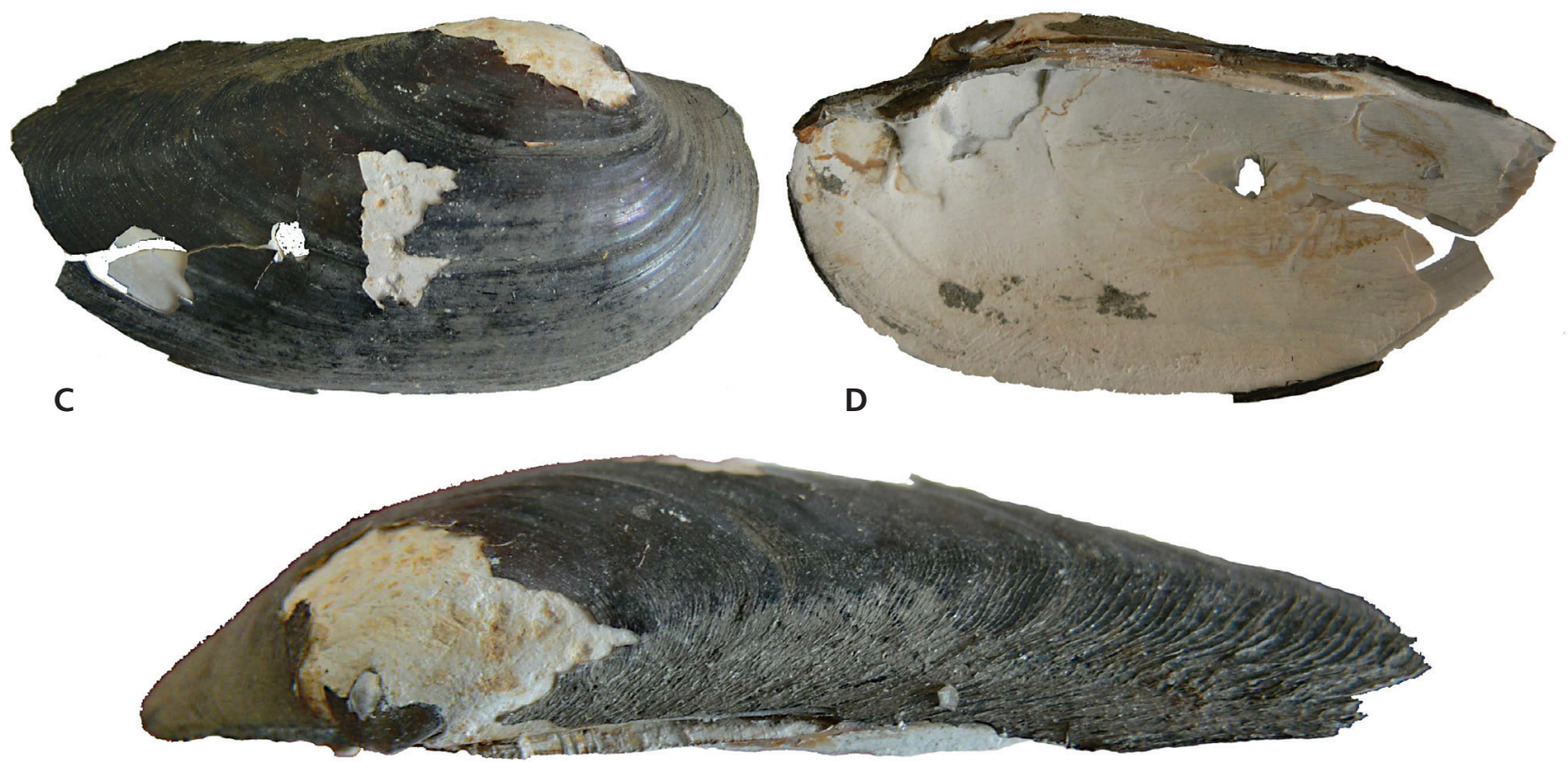

E

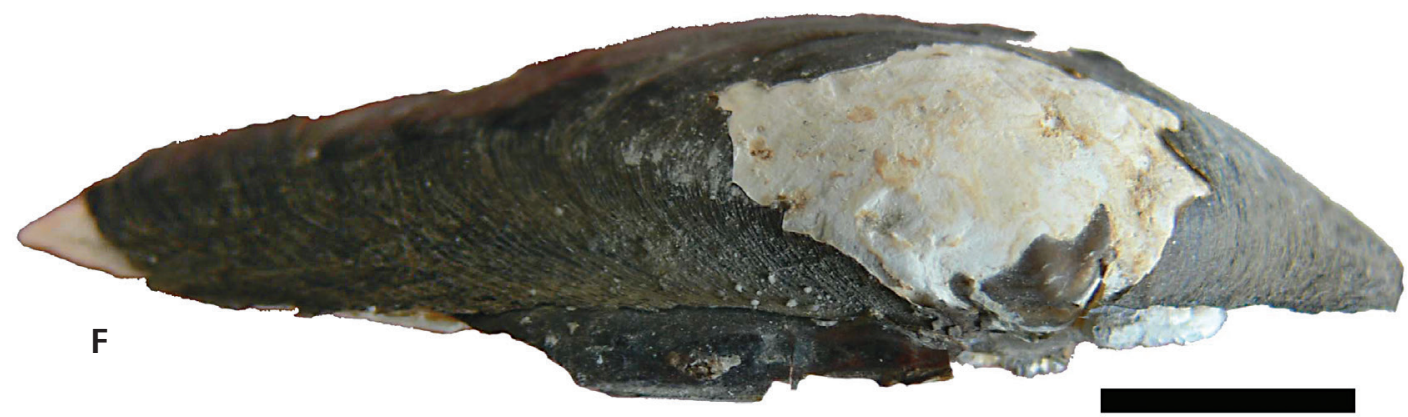

Figure 3. Unio tumidus (specimen UT12-066). A, B - outer and inner shell surfaces of left valve, with severely fragmented posterior end. The inner surface is dull and somewhat chalky. C, D - outer and inner shell surfaces of right valve, with slightly fragmented posterior end. E, F - dorsal view of the right and left valves. The ligament is partly missing, while the umbonal areas are somewhat dissolved. Scale bars $10 \mathrm{~mm}$.

periostracum of $U$. crassus (UC16-005). A thin crust of brown mineralisation appears on a few valves (Fig. 2A, B; Tabs 1, 2).

The taphonomical grade of fragmentation appears mostly from 0 to 1 , whereas the grade of 2 is only rarely obtained (Tab. 3), which may be due to a sampling strategy towards unfragmented valves. The posterior end of the shells is commonly fragmented in a way leading to their irregular appearance. Fragmentation shows no obvious relationship with dorso-ventral height. Instead, an increas- 
Table 1. Shell dimensions and taphonomical features scored for individual valves of Unio crassus. Abbreviations: $\mathrm{V}-\mathrm{valve}(\mathrm{L}=$ left; $\mathrm{R}=$ right $)$; PA - posterior-anterior; DV - dorso-ventral; F - fragmentation; Doss - dissolution outer shell surface; (p0, 1, 2 = partly 0, 1, 2; - = unobservable); Diss - dissolution inner shell surface; Du - dissolution umbo; Lis - lustre inner surface; Dul - dissolution umbo length; Duw - dissolution umbo width; $\mathrm{Pnu}$ - periostracum non-umbo; $\mathrm{Pu}$ - periostracum umbo; Lg - ligament.

\begin{tabular}{|c|c|c|c|c|c|c|c|c|c|c|c|c|c|c|}
\hline Shell No. & $\mathrm{V}$ & $\begin{array}{c}\mathrm{PA} \\
(\mathrm{mm})\end{array}$ & $\begin{array}{l}\text { DV } \\
(\mathrm{mm})\end{array}$ & $\mathrm{F}$ & Doss & Diss & $\mathrm{Du}$ & Lis & $\begin{array}{c}\text { Dul } \\
(\mathrm{mm})\end{array}$ & $\begin{array}{l}\text { Duw } \\
(\mathrm{mm})\end{array}$ & Pnu & $\mathrm{Pu}$ & Lg & Comments \\
\hline UC11-001 & $\mathrm{L}$ & 45 & 26 & 1 & - & 1 & 1 & 1 & 5.24 & 3.13 & 1 & 1 & 2 & Minor fragmented posterior end. \\
\hline UC11-001 & $\mathrm{R}$ & 45 & 26 & 1 & - & 1 & & 1 & & & 1 & & 2 & Minor fragmented posterior end. \\
\hline UC11-002 & $\mathrm{L}$ & 59 & 31 & 1 & - & 0 & 2 & 1 & 12.10 & 7.38 & 0 & 2 & 1 & Scarcely fragmented. \\
\hline UC11-002 & $\mathrm{R}$ & 59 & 31 & 0 & - & 0 & & 1 & & & 0 & & 1 & \\
\hline UC11-003 & $\mathrm{L}$ & 67 & 34 & 0 & - & 0 & 1 & 2 & 6.84 & 3.86 & 0 & 1 & 2 & \\
\hline UC11-003 & $\mathrm{R}$ & 67 & 34 & 0 & - & 0 & 1 & 1 & & 3.96 & 0 & 1 & 2 & \\
\hline UC12-001 & $\mathrm{L}$ & 43 & 24 & 0 & - & 0 & 1 & 1 & 4.00 & 3.49 & 0 & 1 & 1 & \\
\hline UC12-001 & $\mathrm{R}$ & 43 & 24 & 0 & - & 0 & 1 & 1 & 3.43 & & 0 & 1 & 1 & \\
\hline UC12-002 & $\mathrm{L}$ & & 31 & 1 & - & 0 & 2 & 1 & 9.97 & 6.71 & 1 & 2 & 2 & $\begin{array}{l}\text { Minor fragmented positerior end. } \\
\text { Dissolution hole in umbo. }\end{array}$ \\
\hline UC12-002 & $\mathrm{R}$ & & 31 & 1 & - & 1 & 2 & 2 & & 6.59 & 1 & 2 & 2 & $\begin{array}{l}\text { Minor fragmented positerior end. } \\
\text { Dissolution hole in umbo. }\end{array}$ \\
\hline UC12-003 & $\mathrm{L}$ & & 34 & & & & & & & & & & & Valve absent. \\
\hline UC12-003 & $\mathrm{R}$ & & 34 & 1 & - & 1 & 2 & 2 & & 7.53 & 1 & 2 & 2 & Minor fragmented posterior end. \\
\hline UC12-004 & $\mathrm{L}$ & 61 & 32 & 1 & - & 0 & 1 & 1 & 7.86 & 4.98 & 1 & 1 & 2 & Minor fragmented ventro-posterior end. \\
\hline UC12-004 & $\mathrm{R}$ & 61 & 32 & 2 & - & 0 & 1 & 1 & & 5.42 & 2 & 1 & 2 & Major fragmented posterior end. \\
\hline UC12-005 & $\mathrm{L}$ & 68 & 35 & 0 & - & 2 & 1 & 2 & 7.88 & 4.56 & 0 & 1 & 2 & \\
\hline UC12-005 & $\mathrm{R}$ & 68 & 35 & 0 & - & 2 & 1 & 2 & & 4.87 & 0 & 1 & 2 & \\
\hline UC12-006 & $\mathrm{L}$ & & 36 & 1 & - & 1 & 2 & 2 & 12.40 & 7.85 & 0 & 2 & 2 & Minor fragmented posterior end. \\
\hline UC12-006 & $\mathrm{R}$ & & 36 & 1 & - & 1 & 2 & 2 & & 8.54 & 0 & 2 & 2 & Minor fragmented posterior end. \\
\hline UC12-007 & $\mathrm{L}$ & & 41 & 2 & - & 2 & 2 & 2 & & 11.9 & 2 & 2 & 2 & Major fragmented posterior end. \\
\hline UC12-007 & $\mathrm{R}$ & & 41 & 1 & p2 and - & 2 & 2 & 2 & & 12.00 & 2 & 2 & 2 & Major fragmented posterior end. \\
\hline UC12-008 & $\mathrm{L}$ & 79 & 38 & 0 & - & 0 & 1 & 1 & 10.20 & 6.50 & 0 & 1 & 1 & Dissolution hole in umbo. \\
\hline UC12-008 & $\mathrm{R}$ & 79 & 38 & 0 & - & 1 & 1 & 2 & & 7.55 & 0 & 1 & 1 & \\
\hline UC13-001 & $\mathrm{L}$ & 46 & 25 & 0 & - & 0 & 1 & 0 & 5.93 & 3.81 & 0 & 1 & 1 & \\
\hline UC13-001 & $\mathrm{R}$ & 46 & 25 & 1 & - & 0 & 1 & 0 & & 3.68 & 0 & 1 & 1 & Minor posterior end. \\
\hline UC13-002 & $\mathrm{L}$ & & 34 & 1 & p2 and - & 2 & 2 & 2 & & & 2 & 2 & 2 & $\begin{array}{l}\text { Major fragmented posterior end. } \\
\text { Dissolved hole in umbo. Brown } \\
\text { mineralization irregularly distributed } \\
\text { on the external calcarous surface and } \\
\text { posterior periostracum. }\end{array}$ \\
\hline UC13-002 & $\mathrm{R}$ & & 34 & 2 & p2 and - & 2 & 2 & 2 & & 13.5 & 2 & 2 & 2 & $\begin{array}{l}\text { Major fragmented posterior end. } \\
\text { Dissolved hole in umbo. Brown } \\
\text { mineralization irregularly distributed } \\
\text { on the external calcarous surface and } \\
\text { posterior periostracum. }\end{array}$ \\
\hline UC13-003 & $\mathrm{L}$ & & 35 & 0 & - & 0 & 1 & 0 & & & 0 & 1 & 1 & \\
\hline UC13-003 & $\mathrm{R}$ & & 35 & 0 & - & 0 & 1 & 0 & & & 0 & 1 & 1 & \\
\hline UC14-001 & $\mathrm{L}$ & 34 & 19 & 0 & - & 1 & 2 & 1 & 6.27 & 4.42 & 0 & 2 & 2 & Dissolution hole in umbo. \\
\hline UC14-001 & $\mathrm{R}$ & 34 & 19 & 0 & - & 1 & & 1 & & & 0 & & 2 & \\
\hline UC14-002 & $\mathrm{L}$ & 41 & 24 & 1 & - & 0 & 2 & 1 & 6.37 & 4.1 & 1 & 2 & 2 & Minor fragmented posterior end. \\
\hline UC14-002 & $\mathrm{R}$ & 41 & 24 & 1 & - & 1 & 2 & 1 & & & 1 & 1 & 2 & \\
\hline UC14-003 & $\mathrm{L}$ & 51 & 27 & 0 & - & 1 & 2 & 2 & 13.4 & 6.93 & 0 & 2 & 1 & \\
\hline UC14-003 & $\mathrm{R}$ & 51 & 27 & 0 & - & 1 & 2 & 2 & & 7.28 & 0 & 2 & 2 & \\
\hline UC14-004 & $\mathrm{L}$ & & 35 & 1 & p2 and - & 2 & 2 & 2 & 23.5 & 13.2 & 2 & 2 & 2 & $\begin{array}{l}\text { Minor fragmented posterior end. } \\
\text { Dissolution hole in umbo. }\end{array}$ \\
\hline UC14-004 & $\mathrm{R}$ & & 35 & 2 & p2 and - & 2 & 2 & 2 & & 16.8 & 2 & 2 & 2 & $\begin{array}{l}\text { Minor fragmented ends. Dissolution } \\
\text { hole in umbo. }\end{array}$ \\
\hline UC14-005 & $\mathrm{L}$ & & 37 & 1 & p2 and - & 1 & 2 & 2 & 21.9 & 15.2 & 2 & 2 & 2 & Minor fragmented posterior end. \\
\hline UC14-005 & $\mathrm{R}$ & & 37 & 1 & p2 and - & 2 & 2 & 2 & & 16.1 & 2 & 2 & 2 & Minor fragmented posterior end. \\
\hline
\end{tabular}


Table 1. Continued.

\begin{tabular}{|c|c|c|c|c|c|c|c|c|c|c|c|c|c|c|}
\hline Shell No. & $\mathrm{V}$ & $\begin{array}{c}\mathrm{PA} \\
(\mathrm{mm})\end{array}$ & $\begin{array}{l}\mathrm{DV} \\
(\mathrm{mm})\end{array}$ & $\mathrm{F}$ & Doss & Diss & $\mathrm{Du}$ & Lis & $\begin{array}{c}\text { Dul } \\
(\mathrm{mm})\end{array}$ & $\begin{array}{l}\text { Duw } \\
(\mathrm{mm})\end{array}$ & Pnu & $\mathrm{Pu}$ & $\mathrm{Lg}$ & Comments \\
\hline UC15-002 & $\mathrm{L}$ & & 36 & 1 & p1 and - & 0 & & 1 & & & 1 & & 2 & \\
\hline UC15-002 & $\mathrm{R}$ & & 36 & 0 & - & 0 & 1 & 1 & & & 0 & 1 & 2 & \\
\hline UC15-001 & $\mathrm{L}$ & & 32 & 1 & - & 1 & & 2 & & & 1 & & 2 & Minor fragmented posterior end. \\
\hline UC15-001 & $\mathrm{R}$ & & 32 & 0 & - & 1 & 1 & 2 & & 4.81 & 1 & 1 & 2 & \\
\hline UC15-003 & $\mathrm{L}$ & & 36 & 1 & p1 and - & 2 & & 2 & & & 1 & & 1 & Minor fragmented posterior end. \\
\hline UC15-003 & $\mathrm{R}$ & & 36 & 1 & p1 and - & 2 & 1 & 2 & & 5.58 & 1 & 1 & 1 & Minor fragmented posterior end. \\
\hline UC15-004 & $\mathrm{L}$ & & 35 & 0 & - & 1 & & 1 & & & 0 & & 2 & \\
\hline UC15-004 & $\mathrm{R}$ & & 35 & 0 & - & 1 & 1 & 1 & & & 0 & 1 & 1 & \\
\hline UC15-005 & $\mathrm{L}$ & & 42 & 0 & - & 1 & & 1 & & & 0 & & 0 & \\
\hline UC15-005 & $\mathrm{R}$ & & 42 & 0 & - & 1 & & 1 & & & 0 & & 0 & \\
\hline UC16-001 & $\mathrm{L}$ & & 23 & 2 & - & 1 & 2 & 2 & 9.97 & 4.35 & 2 & 2 & 2 & Major fragmented posterior end. \\
\hline UC16-001 & $\mathrm{R}$ & & 23 & 2 & - & 1 & 2 & 2 & & 5.53 & 2 & 2 & 2 & Major fragmented posterior end. \\
\hline UC16-002 & $\mathrm{L}$ & & 26 & 2 & - & 2 & 2 & 2 & 10.20 & 5.86 & 2 & 2 & 2 & Major fragmented posterior end. \\
\hline UC16-002 & $\mathrm{R}$ & & 26 & 2 & - & 1 & 2 & 1 & & 6.47 & 2 & 2 & 2 & Major fragmented posterior end. \\
\hline UC16-003 & $\mathrm{L}$ & 45 & 24 & 0 & - & 0 & 2 & 1 & 7.70 & 4.29 & 0 & 2 & 2 & \\
\hline UC16-003 & $\mathrm{R}$ & 45 & 24 & 0 & - & 0 & 2 & 1 & & 4.57 & 0 & 2 & 2 & \\
\hline UC16-004 & $\mathrm{L}$ & & 29 & 1 & - & 1 & 1 & 1 & 7.62 & 4.18 & 1 & 1 & 2 & Minor fragmented positerior end. \\
\hline UC16-004 & $\mathrm{R}$ & & 29 & 1 & - & 1 & 1 & 1 & & 4.37 & 1 & 1 & 2 & Minor fragmented positerior end. \\
\hline UC16-005 & $\mathrm{L}$ & 50 & 28 & 0 & - & 0 & 1 & 1 & 8.73 & 5.97 & 0 & 2 & 2 & $\begin{array}{l}\text { Brown mineralization on the } \\
\text { periostracum at the posterior end and } \\
\text { towards the umbo. Houses of caddis } \\
\text { larvae on dorso-posterior part. }\end{array}$ \\
\hline UC16-005 & $\mathrm{R}$ & 50 & 28 & 0 & - & 0 & & 1 & & & 0 & & 2 & $\begin{array}{l}\text { Brown mineralization on the } \\
\text { periostracum at the posterior end and } \\
\text { towards the umbo. Houses of caddis } \\
\text { larvae on dorso-posterior part. }\end{array}$ \\
\hline UC16-006 & $\mathrm{L}$ & & 37 & 1 & p1 and - & 1 & 2 & 1 & 14.2 & 8.54 & 2 & 2 & 2 & $\begin{array}{l}\text { Minor fragmented positerior end. } \\
\text { Dissolution hole in umbo. }\end{array}$ \\
\hline UC16-006 & $\mathrm{R}$ & & 37 & 2 & - & 1 & 2 & 1 & & 8.44 & 2 & 2 & 2 & $\begin{array}{l}\text { Major fragmented posterior end. } \\
\text { Dissolution hole in umbo. }\end{array}$ \\
\hline UC16-007 & $\mathrm{L}$ & 70 & 35 & 0 & - & 1 & 1 & 2 & 12.00 & 7.01 & 0 & 1 & 1 & \\
\hline UC16-007 & $\mathrm{R}$ & 70 & 35 & 1 & - & 1 & 1 & 2 & & 7.36 & 1 & 1 & 2 & Minor fragmented posterior end. \\
\hline UC16-008 & $\mathrm{L}$ & 72 & 36 & 0 & - & 2 & 1 & 2 & 10.10 & 6.90 & 0 & 1 & 2 & \\
\hline UC16-008 & $\mathrm{R}$ & 72 & 36 & 0 & - & 2 & 1 & 2 & & 7.69 & 0 & 1 & 2 & \\
\hline UC16-009 & $\mathrm{L}$ & 73 & 37 & 0 & - & 2 & 2 & 1 & 12.10 & 9.55 & 0 & 2 & 2 & \\
\hline UC16-009 & $\mathrm{R}$ & 73 & 37 & 0 & - & 1 & 2 & 1 & 7.910 & & 0 & 2 & 1 & \\
\hline UC17-001 & $\mathrm{L}$ & 69 & 36 & 1 & - & 0 & 2 & 0 & 18.80 & 13.90 & 0 & 2 & 0 & Minor fragmented posterior end. \\
\hline UC17-001 & $\mathrm{R}$ & 69 & 36 & 0 & - & 0 & 2 & 0 & 17.10 & 12.50 & 0 & 2 & 0 & Dissolution hole at anterior muscle scar. \\
\hline UC17-002 & $\mathrm{L}$ & 82 & 43 & 1 & p1 and - & 1 & 2 & 2 & 22.90 & 12.90 & 1 & 2 & 2 & $\begin{array}{l}\text { Minor fragmented posterior end. } \\
\text { Brown mineralization on the } \\
\text { periostracum at the posterior end } \\
\text { and towards the umbo. }\end{array}$ \\
\hline UC17-002 & $\mathrm{R}$ & 82 & 43 & 0 & - & 1 & 2 & 2 & 19.80 & 11.80 & 0 & 2 & 1 & $\begin{array}{l}\text { Brown mineralization on the } \\
\text { periostracum at the posterior end } \\
\text { and towards the umbo. }\end{array}$ \\
\hline UC19-001 & $\mathrm{L}$ & 36 & 20 & 0 & - & 0 & 1 & 1 & 6.79 & 4.38 & 0 & 2 & 1 & \\
\hline UC19-001 & $\mathrm{R}$ & 36 & 20 & 0 & - & 0 & 1 & 1 & & 4.27 & 0 & 2 & 1 & \\
\hline UC19-002 & $\mathrm{L}$ & 46 & 27 & 0 & - & 1 & 2 & 2 & 8.87 & 4.50 & 0 & 2 & 2 & \\
\hline UC19-002 & $\mathrm{R}$ & 46 & 27 & 0 & - & 2 & 2 & 2 & & 5.19 & 0 & 2 & 2 & \\
\hline UC20-001 & $\mathrm{L}$ & 83 & 44 & 0 & - & 0 & 2 & 0 & 14.50 & 7.48 & 0 & 2 & 0 & \\
\hline UC20-001 & $\mathrm{R}$ & 83 & 44 & 0 & - & 0 & 2 & 0 & 17.20 & 9.78 & 0 & 2 & 0 & \\
\hline UC20-002 & $\mathrm{L}$ & 77 & 36 & 0 & - & 0 & 2 & 2 & 14.60 & 7.68 & 0 & 2 & 1 & \\
\hline UC20-002 & $\mathrm{R}$ & 77 & 36 & 0 & - & 1 & 2 & 2 & 12.50 & 8.03 & 0 & 2 & 1 & \\
\hline
\end{tabular}


Table 2. Shell dimensions and taphonomical features scored for individual valves of Unio tumidus. Abbreviations: $\mathrm{V}-\mathrm{valve}(\mathrm{L}=$ left; $\mathrm{R}=\mathrm{right})$; PA - posterior-anterior; DV - dorso-ventral; F - fragmentation; Doss - dissolution outer shell surface; (p0, 1, 2= partly 0, 1, 2; - = unobservable); Diss - dissolution inner shell surface; Du - dissolution umbo; Lis - lustre inner surface; Dul - dissolution umbo length; Duw - dissolution umbo width; $\mathrm{Pnu}$ - periostracum non-umbo; $\mathrm{Pu}$ - periostracum umbo; Lg - ligament.

\begin{tabular}{|c|c|c|c|c|c|c|c|c|c|c|c|c|c|c|}
\hline Shell No. & $\mathrm{V}$ & $\begin{array}{c}\mathrm{PA} \\
(\mathrm{mm})\end{array}$ & $\begin{array}{c}\text { DV } \\
(\mathrm{mm})\end{array}$ & $\mathrm{F}$ & Doss & Diss & $\mathrm{Du}$ & Lis & $\begin{array}{c}\text { Dul } \\
(\mathrm{mm})\end{array}$ & $\begin{array}{l}\text { Duw } \\
\text { (mm) }\end{array}$ & Pnu & $\mathrm{Pu}$ & $\mathrm{Lg}$ & Comments \\
\hline UT12-001 & $\mathrm{L}$ & 61.2 & 28.8 & 2 & - & 0 & 1 & 1 & 7.2 & 5.8 & 2 & 1 & 0 & Major fragmented posterior end. \\
\hline UT12-001 & $\mathrm{R}$ & 61.2 & 28.8 & 0 & - & 0 & 1 & 1 & 8.6 & 5.7 & 0 & 1 & 0 & \\
\hline UT12-002 & $\mathrm{L}$ & 61.8 & 30.0 & 1 & - & 0 & 1 & 1 & 8.0 & 5.0 & 1 & 1 & 0 & Minor fragmented posterior end. \\
\hline UT12-002 & $\mathrm{R}$ & 61.8 & 30.0 & 1 & $\mathrm{p} 0$ and - & 1 & 1 & 2 & 5.2 & 4.8 & 1 & 1 & 0 & Minor fragmented posterior end. \\
\hline UT12-003 & $\mathrm{L}$ & 59.9 & 29.5 & 0 & - & 0 & 2 & 0 & 11.0 & 7.5 & 0 & 2 & 1 & \\
\hline UT12-003 & $\mathrm{R}$ & 59.9 & 29.5 & 0 & - & 0 & 2 & 1 & 13.5 & 7.2 & 0 & 2 & 1 & \\
\hline UT12-004 & $\mathrm{L}$ & & 27.1 & 1 & p1 and - & 0 & 2 & 1 & 9.8 & 6.7 & 1 & 2 & 0 & Minor fragmented posterior end. \\
\hline UT12-004 & $\mathrm{R}$ & & 27.1 & 2 & p0 and - & 0 & 2 & 1 & 11.0 & 6.4 & 2 & 2 & 0 & Major fragmented posterior end. \\
\hline UT12-005 & $\mathrm{L}$ & & 27.5 & 2 & - & 1 & 2 & 2 & 10.3 & 8.1 & 2 & 2 & 1 & Major fragmented posterior end. \\
\hline UT12-005 & $\mathrm{R}$ & & 27.5 & 1 & - & 1 & 1 & 2 & 8.5 & 6.6 & 1 & 2 & 1 & Minor fragmented posterior end. \\
\hline UT12-006 & $\mathrm{L}$ & & 37.0 & 2 & p1 and - & 2 & 2 & 2 & 17.1 & 12.0 & 2 & 2 & 0 & Minor fragmented posterior end. \\
\hline UT12-006 & $\mathrm{R}$ & & 37.0 & 2 & p1 and - & 2 & 2 & 2 & 17.2 & 11.6 & 2 & 2 & 0 & Major fragmented posterior end. \\
\hline UT12-007 & $\mathrm{L}$ & 61.7 & 29.2 & 0 & - & 0 & 1 & 1 & 10.1 & 5.5 & 0 & 1 & 0 & \\
\hline UT12-007 & $\mathrm{R}$ & 61.7 & 29.2 & 0 & - & 1 & 1 & 2 & 11.2 & 6.6 & 0 & 1 & 0 & \\
\hline UT12-008 & $\mathrm{L}$ & 60.9 & 30.0 & 0 & - & 1 & 1 & 1 & 6.1 & 6.6 & 0 & 1 & 0 & \\
\hline UT12-008 & $\mathrm{R}$ & 60.9 & 30.0 & 0 & - & 1 & 1 & 1 & 6.4 & 4.9 & 0 & 1 & 0 & \\
\hline UT12-009 & $\mathrm{L}$ & 50.7 & 26.1 & 0 & p2 and - & 1 & 2 & 2 & 10.9 & 6.2 & 1 & 2 & 0 & \\
\hline UT12-009 & $\mathrm{R}$ & 50.7 & 26.1 & 0 & - & 1 & 2 & 2 & 10.6 & 6.0 & 0 & 2 & 0 & \\
\hline UT12-010 & $\mathrm{L}$ & 45.6 & 23.0 & 0 & - & 0 & 2 & 0 & 8.4 & 8.1 & 0 & 2 & 0 & \\
\hline UT12-010 & $\mathrm{R}$ & 45.6 & 23.0 & 0 & - & 0 & 2 & 0 & 8.6 & 8.2 & 0 & 2 & 0 & \\
\hline UT12-011 & $\mathrm{L}$ & & 32.4 & 1 & - & 0 & 2 & 2 & 13.6 & 7.8 & 1 & 2 & 0 & Minor fragmented posterior end. \\
\hline UT12-011 & $\mathrm{R}$ & & 32.4 & 1 & - & 0 & 2 & 2 & 14.7 & 11.3 & 1 & 2 & 0 & Minor fragmented posterior end. \\
\hline UT12-012 & $\mathrm{L}$ & 62.5 & 31.3 & 0 & - & 0 & 1 & 2 & 6.0 & 4.1 & 0 & 1 & 0 & \\
\hline UT12-012 & $\mathrm{R}$ & 62.5 & 31.3 & 0 & - & 0 & 1 & 2 & 5.3 & 4.4 & 0 & 1 & 0 & \\
\hline UT12-013 & $\mathrm{L}$ & 57.4 & 26.8 & 0 & - & 0 & 1 & 0 & 10.4 & 4.3 & 0 & 1 & 0 & \\
\hline UT12-013 & $\mathrm{R}$ & 57.4 & 26.8 & 0 & - & 0 & 2 & 0 & 8.6 & 5.6 & 0 & 2 & 0 & \\
\hline UT12-014 & $\mathrm{L}$ & 57.6 & 28.3 & 1 & $\mathrm{p} 0$ and - & 0 & 1 & 2 & 7.8 & 5.7 & 1 & 1 & 0 & Hole in central area. \\
\hline UT12-014 & $\mathrm{R}$ & 57.6 & 28.3 & 0 & - & 0 & 2 & 2 & 8.5 & 6.1 & 0 & 2 & 0 & \\
\hline UT12-015 & $\mathrm{L}$ & 58.2 & 29.4 & 1 & - & 1 & 2 & 2 & 12.5 & 10.9 & 1 & 2 & 0 & Minor fragmented posterior end. \\
\hline UT12-015 & $\mathrm{R}$ & 58.2 & 29.4 & 0 & p1 and - & 1 & 2 & 2 & 12.2 & 10.8 & 1 & 2 & 0 & \\
\hline UT12-016 & $\mathrm{L}$ & 52.3 & 26.0 & 1 & - & 0 & 2 & 1 & 7.7 & 6.0 & 1 & 2 & 0 & Scarcely fragmented. \\
\hline UT12-016 & $\mathrm{R}$ & 52.3 & 26.0 & 0 & - & 0 & 2 & 0 & 8.3 & 6.0 & 0 & 2 & 0 & \\
\hline UT12-017 & $\mathrm{L}$ & & 31.3 & 1 & - & 0 & 2 & 0 & 8.3 & 5.6 & 1 & 2 & 0 & Minor fragmented posterior end. \\
\hline UT12-017 & $\mathrm{R}$ & & 31.3 & 1 & $\mathrm{p} 0$ and - & 0 & 2 & 0 & 8.6 & 5.4 & 1 & 2 & 0 & Minor fragmented posterior end. \\
\hline UT12-018 & $\mathrm{L}$ & 55.2 & 27.7 & 0 & - & 0 & 2 & 1 & 9.2 & 6.2 & 0 & 2 & 0 & \\
\hline UT12-018 & $\mathrm{R}$ & 55.2 & 27.7 & 0 & - & 0 & 2 & 0 & 8.9 & 7.9 & 0 & 2 & 0 & \\
\hline UT12-019 & $\mathrm{L}$ & 76.9 & 35.3 & 1 & - & 0 & 2 & 2 & 13.6 & 11.3 & 1 & 2 & 0 & Minor fragmented posterior end. \\
\hline UT12-019 & $\mathrm{R}$ & 76.9 & 35.3 & 0 & - & 0 & 2 & 2 & 15.8 & 13.4 & 0 & 2 & 0 & \\
\hline UT12-020 & $\mathrm{L}$ & 40.3 & 21.4 & 0 & - & 1 & 2 & 2 & 10.7 & 7.6 & 0 & 2 & 0 & \\
\hline UT12-020 & $\mathrm{R}$ & 40.3 & 21.4 & 1 & - & 1 & 2 & 2 & 9.5 & 10.8 & 1 & 2 & 0 & Minor fragmented posterior end. \\
\hline UT12-021 & $\mathrm{L}$ & 65.0 & 32.0 & 0 & - & 2 & 1 & 2 & 6.8 & 4.8 & 0 & 1 & 0 & Some organic sheet inside the valves. \\
\hline UT12-021 & $\mathrm{R}$ & 65.0 & 32.0 & 0 & - & 2 & 1 & 2 & 7.5 & 5.1 & 0 & 1 & 0 & \\
\hline UT12-022 & $\mathrm{L}$ & 77.8 & 35.4 & 1 & $\mathrm{p} 0$ and - & 1 & 2 & 2 & 13.6 & 9.4 & 1 & 2 & 0 & Minor fragmented posterior end. \\
\hline UT12-022 & $\mathrm{R}$ & 77.8 & 35.4 & 1 & p0 and - & 1 & 1 & 2 & 11.2 & 7.3 & 1 & 1 & 0 & Minor fragmented posterior end. \\
\hline UT12-023 & $\mathrm{L}$ & & 25.9 & 2 & - & 1 & 1 & 2 & 8.9 & 5.8 & 2 & 1 & 0 & Major fragmented posterior end. \\
\hline UT12-023 & $\mathrm{R}$ & & 25.9 & 1 & - & 1 & 1 & 2 & 9.0 & 4.9 & 1 & 1 & 0 & Minor fragmented posterior end. \\
\hline UT12-024 & $\mathrm{L}$ & 70.6 & 33.3 & 0 & - & 0 & 1 & 0 & 7.1 & 5.9 & 0 & 1 & 0 & \\
\hline UT12-024 & $\mathrm{R}$ & 70.6 & 33.3 & 1 & - & 0 & 1 & 1 & 6.4 & 5.2 & 0 & 1 & 0 & Minor fragmented posterior end. \\
\hline
\end{tabular}


Table 2. Continued.

\begin{tabular}{|c|c|c|c|c|c|c|c|c|c|c|c|c|c|c|}
\hline Shell No. & $\mathrm{V}$ & $\begin{array}{c}\mathrm{PA} \\
(\mathrm{mm})\end{array}$ & $\begin{array}{l}\mathrm{DV} \\
(\mathrm{mm})\end{array}$ & $\mathrm{F}$ & Doss & Diss & $\mathrm{Du}$ & Lis & $\begin{array}{c}\text { Dul } \\
(\mathrm{mm})\end{array}$ & $\begin{array}{l}\text { Duw } \\
(\mathrm{mm})\end{array}$ & Pnu & $\mathrm{Pu}$ & $\mathrm{Lg}$ & Comments \\
\hline UT12-025 & $\mathrm{L}$ & 64.5 & 31.7 & 0 & - & 0 & 1 & 0 & 8.6 & 8.3 & 0 & 1 & 0 & \\
\hline UT12-025 & $\mathrm{R}$ & 64.5 & 31.7 & 0 & - & 0 & 1 & 0 & 7.9 & 5.7 & 0 & 1 & 0 & \\
\hline UT12-026 & $\mathrm{L}$ & 63.2 & 30.0 & 2 & - & 0 & 2 & 2 & 11.5 & 7.3 & 2 & 2 & 0 & Major fragmented posterior end. \\
\hline UT12-026 & $\mathrm{R}$ & 63.2 & 30.0 & 0 & - & 0 & 2 & 2 & 12.7 & 8.3 & 0 & 2 & 0 & \\
\hline UT12-027 & $\mathrm{L}$ & 57.4 & 28.2 & 0 & - & 0 & 2 & 2 & 13.4 & 10.0 & 0 & 2 & 0 & \\
\hline UT12-027 & $\mathrm{R}$ & 57.4 & 28.2 & 1 & $\mathrm{p} 0$ and - & 0 & 2 & 2 & 17.9 & 10.2 & 1 & 2 & 0 & Minor fragmented posterior end. \\
\hline UT12-028 & $\mathrm{L}$ & 59.9 & 28.6 & 0 & - & 1 & 1 & 1 & 7.7 & 4.9 & 0 & 1 & 0 & \\
\hline UT12-028 & $\mathrm{R}$ & 59.9 & 28.6 & 0 & - & 1 & 1 & 1 & 8.3 & 5.3 & 0 & 1 & 0 & \\
\hline UT12-029 & $\mathrm{L}$ & 66.9 & 33.4 & 2 & - & 1 & 2 & 2 & 13.0 & 9.4 & 2 & 2 & 0 & Major fragmented posterior end. \\
\hline UT12-029 & $\mathrm{R}$ & 66.9 & 33.4 & 0 & - & 1 & 2 & 2 & 11.9 & 11.3 & 1 & 2 & 0 & \\
\hline UT12-030 & $\mathrm{L}$ & 53.4 & 26.6 & 0 & - & 1 & 2 & 1 & 9.1 & 5.9 & 0 & 2 & 0 & \\
\hline UT12-030 & $\mathrm{R}$ & 53.4 & 26.6 & 0 & - & 1 & 2 & 1 & 8.6 & 6.7 & 0 & 2 & 0 & \\
\hline UT12-031 & $\mathrm{L}$ & & 31.1 & 1 & p1 and - & 2 & 2 & 2 & 10.8 & 6.8 & 1 & 2 & 0 & Minor fragmented posterior end. \\
\hline UT12-031 & $\mathrm{R}$ & & 31.1 & 1 & p1 and- & 2 & 2 & 2 & 13.6 & 9.7 & 1 & 2 & 0 & Minor fragmented posterior end. \\
\hline UT12-032 & $\mathrm{L}$ & 63.2 & 31.2 & 1 & - & 1 & 2 & 2 & 10.3 & 6.9 & 1 & 2 & 1 & Clipping along post-ventral margin. \\
\hline UT12-032 & $\mathrm{R}$ & 63.2 & 31.2 & 1 & - & 1 & 2 & 2 & 10.6 & 7.4 & 1 & 2 & 1 & Clipping along post-ventral margin. \\
\hline UT12-033 & $\mathrm{L}$ & 64.7 & 32.3 & 1 & p0 and - & 0 & 1 & 1 & 7.8 & 5.6 & 1 & 1 & 0 & Minor fragmented posterior end. \\
\hline UT12-033 & $\mathrm{R}$ & 64.7 & 32.3 & 1 & p0 and - & 0 & 1 & 1 & 7.1 & 5.9 & 1 & 1 & 0 & Minor fragmented posterior end. \\
\hline UT12-034 & $\mathrm{L}$ & 50.4 & 25.4 & 0 & - & 0 & 2 & 2 & 10.3 & 7.8 & 0 & 2 & 0 & \\
\hline UT12-034 & $\mathrm{R}$ & 50.4 & 25.4 & 0 & - & 0 & 2 & 2 & 12.3 & 8.3 & 0 & 2 & 0 & \\
\hline UT12-035 & $\mathrm{L}$ & & 30.3 & 1 & - & 0 & 2 & 1 & 11.8 & 6.6 & 1 & 2 & 2 & Minor fragmented posterior end. \\
\hline UT12-035 & $\mathrm{R}$ & & 30.3 & 1 & - & 0 & 2 & 1 & 11.2 & 7.2 & 1 & 2 & 2 & Minor fragmented posterior end. \\
\hline UT12-036 & $\mathrm{L}$ & 73.1 & 35.1 & 0 & - & 0 & 2 & 1 & 16.0 & 9.8 & 0 & 2 & 1 & \\
\hline UT12-036 & $\mathrm{R}$ & 73.1 & 35.1 & 1 & p1 and - & 0 & 2 & 1 & 19.3 & 13.6 & 1 & 2 & 1 & Minor fragmented posterior end. \\
\hline UT12-037 & $\mathrm{L}$ & 55.3 & 26.8 & 1 & p1 and - & 1 & 2 & 2 & 8.2 & 4.9 & 1 & 2 & 0 & Minor fragmented posterior end. \\
\hline UT12-037 & $\mathrm{R}$ & 55.3 & 26.8 & 1 & p1 and - & 1 & 2 & 2 & 9.0 & 6.0 & 2 & 2 & 0 & Major fragmented posterior end. \\
\hline UT12-038 & $\mathrm{L}$ & & 32.9 & 1 & p2 and - & 1 & 2 & 1 & 18.1 & 13.4 & 1 & 2 & 2 & Minor fragmented posterior end. \\
\hline UT12-038 & $\mathrm{R}$ & & 32.9 & 1 & p2 and - & 1 & 2 & 1 & 16.4 & 8.4 & 1 & 2 & 2 & Minor fragmented posterior end. \\
\hline UT12-039 & $\mathrm{L}$ & 53.1 & 28.7 & 0 & - & 0 & 1 & 1 & 7.4 & 5.6 & 0 & 1 & 0 & \\
\hline UT12-039 & $\mathrm{R}$ & 53.1 & 28.7 & 1 & - & 0 & 1 & 1 & 8.3 & 5.1 & 1 & 1 & 0 & Minor fragmented posterior end. \\
\hline UT12-040 & $\mathrm{L}$ & & 31.7 & 1 & $\mathrm{p} 0$ and - & 0 & 2 & 1 & 11.8 & 8.5 & 1 & 2 & 0 & Minor fragmented posterior end. \\
\hline UT12-040 & $\mathrm{R}$ & & 31.7 & 2 & p1 and - & 0 & 2 & 1 & 13.5 & 8.9 & 2 & 2 & 0 & Major fragmented posterior end. \\
\hline UT12-041 & $\mathrm{L}$ & & 33.6 & 1 & - & 1 & 2 & 2 & 12.3 & 7.1 & 1 & 2 & 0 & Minor fragmented posterior end. \\
\hline UT12-041 & $\mathrm{R}$ & & 33.6 & 1 & p0 and - & 1 & 2 & 2 & 12.3 & 7.9 & 1 & 2 & 0 & Minor fragmented posterior end. \\
\hline UT12-042 & $\mathrm{L}$ & 64.2 & 29.8 & 0 & - & 0 & 1 & 2 & 7.9 & 5.4 & 0 & 1 & 0 & \\
\hline UT12-042 & $\mathrm{R}$ & 64.2 & 29.8 & 1 & p0 and - & 0 & 1 & 2 & 8.0 & 4.2 & 1 & 1 & 0 & Minor fragmented posterior end. \\
\hline UT12-043 & $\mathrm{L}$ & 65.1 & 30.3 & 1 & p0 and - & 1 & 1 & 2 & 9.1 & 7.7 & 1 & 1 & 0 & Minor fragmented posterior end. \\
\hline UT12-043 & $\mathrm{R}$ & 65.1 & 30.3 & 0 & - & 1 & 1 & 2 & 9.8 & 8.1 & 0 & 1 & 0 & \\
\hline UT12-044 & $\mathrm{L}$ & 45.8 & 21.9 & 0 & - & 0 & 2 & 2 & 11.8 & 7.1 & 0 & 2 & 1 & \\
\hline UT12-044 & $\mathrm{R}$ & 45.8 & 21.9 & 1 & - & 0 & 2 & 2 & 11.2 & 6.1 & 0 & 2 & 1 & Minor fragmented posterior end. \\
\hline UT12-045 & $\mathrm{L}$ & 66.9 & 32.3 & 2 & - & 1 & 2 & 2 & 16.3 & 9.2 & 2 & 2 & 0 & $\begin{array}{l}\text { Major fragmented posterior end. Some } \\
\text { organic sheet inside the valves. }\end{array}$ \\
\hline UT12-045 & $\mathrm{R}$ & 66.9 & 32.3 & 1 & - & 1 & 2 & 2 & 13.6 & 8.5 & 1 & 2 & 0 & Minor fragmented posterior end. \\
\hline UT12-046 & $\mathrm{L}$ & 58.7 & 28.4 & 1 & p0 and - & 1 & 2 & 1 & 13.8 & 10.1 & 1 & 2 & 0 & Minor fragmented posterior end. \\
\hline UT12-046 & $\mathrm{R}$ & 58.7 & 28.4 & 0 & p1 and - & 1 & 2 & 1 & 12.2 & 9.1 & 1 & 2 & 0 & \\
\hline UT12-047 & $\mathrm{L}$ & 72.1 & 33.2 & 0 & p1 and - & 1 & 0 & 2 & 14.0 & 10.3 & 0 & 2 & 0 & \\
\hline UT12-047 & $\mathrm{R}$ & 72.1 & 33.2 & 0 & $\mathrm{p} 1$ and - & 1 & 0 & 2 & 12.2 & 8.3 & 0 & 2 & 0 & \\
\hline UT12-048 & $\mathrm{L}$ & 57.1 & 28.9 & 2 & p1 and - & 1 & 2 & 2 & 11.5 & 9.4 & 2 & 2 & 1 & Major fragmented posterior end. \\
\hline UT12-048 & $\mathrm{R}$ & 57.1 & 28.9 & 1 & p1 and - & 1 & 2 & 2 & 18.7 & 10.7 & 1 & 2 & 1 & Minor fragmented posterior end. \\
\hline UT12-049 & $\mathrm{L}$ & 60.7 & 29.1 & 0 & - & 0 & 1 & 2 & 9.2 & 7.4 & 0 & 1 & 0 & \\
\hline UT12-049 & $\mathrm{R}$ & 60.7 & 29.1 & 0 & - & 0 & 2 & 2 & 14.1 & 7.8 & 0 & 2 & 0 & \\
\hline
\end{tabular}


Table 2. Continued.

\begin{tabular}{|c|c|c|c|c|c|c|c|c|c|c|c|c|c|c|}
\hline Shell No. & $\mathrm{V}$ & $\begin{array}{c}\mathrm{PA} \\
(\mathrm{mm})\end{array}$ & $\begin{array}{l}\mathrm{DV} \\
(\mathrm{mm})\end{array}$ & $\mathrm{F}$ & Doss & Diss & $\mathrm{Du}$ & Lis & $\begin{array}{l}\text { Dul } \\
(\mathrm{mm})\end{array}$ & $\begin{array}{l}\text { Duw } \\
(\mathrm{mm})\end{array}$ & Pnu & $\mathrm{Pu}$ & $\mathrm{Lg}$ & Comments \\
\hline UT12-050 & $\mathrm{L}$ & 63.9 & 30.0 & 0 & - & 0 & 1 & 1 & 6.6 & 5.7 & 0 & 1 & 0 & \\
\hline UT12-050 & $\mathrm{R}$ & 63.9 & 30.0 & 0 & - & 0 & 1 & 1 & 6.7 & 5.2 & 0 & 1 & 0 & \\
\hline UT12-051 & $\mathrm{L}$ & & 27.3 & 1 & - & 0 & 2 & 1 & 8.3 & 7.5 & 1 & 2 & 1 & Minor fragmented posterior end. \\
\hline UT12-051 & $\mathrm{R}$ & & 27.3 & 1 & - & 0 & 2 & 1 & 9.1 & 6.7 & 1 & 2 & 1 & Minor fragmented posterior end. \\
\hline UT12-052 & $\mathrm{L}$ & 46.1 & 23.1 & 0 & - & 2 & 1 & 2 & 6.9 & 5.2 & 0 & 1 & 0 & \\
\hline UT12-052 & $\mathrm{R}$ & 46.1 & 23.1 & 0 & - & 1 & 1 & 2 & 6.8 & 4.4 & 0 & 1 & 0 & \\
\hline UT12-053 & $\mathrm{L}$ & 61.2 & 29.7 & 0 & - & 2 & 2 & 2 & 11.5 & 7.5 & 0 & 2 & 0 & Deformations on inside of valves. \\
\hline UT12-053 & $\mathrm{R}$ & 61.2 & 29.7 & 0 & - & 2 & 2 & 2 & 12.5 & 9.9 & 0 & 2 & 0 & Deformations on inside of valves. \\
\hline UT12-054 & $\mathrm{L}$ & 54.2 & 27.0 & 1 & - & 0 & 2 & 1 & 9.0 & 6.5 & 1 & 2 & 1 & Minor fragmented posterior end. \\
\hline UT12-054 & $\mathrm{R}$ & 54.2 & 27.0 & 1 & p1 and - & 0 & 2 & 1 & 10.3 & 7.6 & 1 & 2 & 1 & Minor fragmented posterior end. \\
\hline UT12-055 & $\mathrm{L}$ & 68.0 & 33.2 & 0 & $\mathrm{p} 2$ and - & 1 & 2 & 1 & 10.8 & 8.0 & 1 & 2 & 1 & \\
\hline UT12-055 & $\mathrm{R}$ & 68.0 & 33.2 & 1 & p2 and - & 1 & 2 & 2 & 12.2 & 8.8 & 1 & 2 & 1 & Minor fragmented posterior end. \\
\hline UT12-056 & $\mathrm{L}$ & 49.9 & 26.1 & 1 & $\mathrm{p} 2$ and - & 0 & 2 & 0 & 9.5 & 5.3 & 1 & 2 & 1 & $\begin{array}{l}\text { Minor fragmented posterior end. Minor } \\
\text { brown mineralization on internal shell } \\
\text { surface. }\end{array}$ \\
\hline UT12-056 & $\mathrm{R}$ & 49.9 & 26.1 & 1 & - & 0 & 2 & 1 & 9.6 & 5.8 & 0 & 2 & 1 & $\begin{array}{l}\text { Minor fragmented posterior end. Major } \\
\text { brown mineralization on internal shell } \\
\text { surface. }\end{array}$ \\
\hline UT12-057 & $\mathrm{L}$ & 45.2 & 24.0 & 0 & p1 and - & 2 & 2 & 2 & 7.7 & 6.5 & 0 & 2 & 0 & Deformations on inner surface? \\
\hline UT12-057 & $\mathrm{R}$ & 45.2 & 24.0 & 0 & p1 and - & 2 & 2 & 2 & 7.1 & 5.9 & 0 & 2 & 0 & Deformations on inner surface? \\
\hline UT12-058 & $\mathrm{L}$ & & 31.3 & 1 & p0 and - & 1 & 2 & 2 & 13.3 & 9.1 & 1 & 2 & 0 & Minor fragmented posterior end. \\
\hline UT12-058 & $\mathrm{R}$ & & 31.3 & 2 & p1 and - & 1 & 2 & 2 & 12.7 & 8.9 & 2 & 2 & 0 & Major fragmented posterior end. \\
\hline UT12-059 & $\mathrm{L}$ & & 27.1 & 2 & - & 1 & 2 & 2 & 13.9 & 8.5 & 2 & 2 & 0 & Major fragmented posterior end. \\
\hline UT12-059 & $\mathrm{R}$ & & 27.1 & 2 & - & 1 & 2 & 2 & 13.0 & 7.2 & 2 & 2 & 0 & Major fragmented posterior end. \\
\hline UT12-060 & $\mathrm{L}$ & & 29.9 & 1 & p0 and - & 0 & 2 & 1 & 11.4 & 10.1 & 1 & 2 & 0 & Minor fragmented posterior end. \\
\hline UT12-060 & $\mathrm{R}$ & & 29.9 & 1 & - & 0 & 2 & 1 & 12.2 & 9.0 & 1 & 2 & 0 & Minor fragmented posterior end. \\
\hline UT12-061 & $\mathrm{L}$ & & 30.8 & 2 & $\mathrm{p} 1$ and - & 1 & 2 & 2 & 14.2 & 12.3 & 2 & 2 & 0 & Major fragmented posterior end. \\
\hline UT12-061 & $\mathrm{R}$ & & 30.8 & 1 & p0 and - & 1 & 2 & 2 & 14.2 & 9.6 & 1 & 2 & 0 & Minor fragmented posterior end. \\
\hline UT12-062 & $\mathrm{L}$ & 60.6 & 29.9 & 0 & - & 0 & 1 & 1 & 7.2 & 3.9 & 0 & 1 & 0 & \\
\hline UT12-062 & $\mathrm{R}$ & 60.6 & 29.9 & 2 & - & 0 & 2 & 1 & 9.2 & 7.2 & 2 & 2 & 0 & Major fragmented posterior end. \\
\hline UT12-063 & $\mathrm{L}$ & 72.1 & 34.7 & 0 & - & 0 & 1 & 1 & 8.4 & 7.4 & 0 & 1 & 1 & \\
\hline UT12-063 & $\mathrm{R}$ & 72.1 & 34.7 & 0 & - & 0 & 1 & 1 & 8.3 & 5.5 & 0 & 1 & 1 & \\
\hline UT12-064 & $\mathrm{L}$ & & 28.4 & 1 & p0 and - & 0 & 2 & 1 & 12.7 & 9.1 & 2 & 2 & 1 & Minor fragmented posterior end. \\
\hline UT12-064 & $\mathrm{R}$ & & 28.4 & 1 & $\mathrm{p} 0$ and - & 0 & 2 & 1 & 12.1 & 9.1 & 2 & 2 & 0 & Minor fragmented posterior end. \\
\hline UT12-065 & $\mathrm{L}$ & 55.1 & 26.8 & 0 & - & 0 & 1 & 0 & 7.2 & 4.0 & 0 & 1 & 1 & \\
\hline UT12-065 & $\mathrm{R}$ & 55.1 & 26.8 & 0 & - & 0 & 1 & 0 & 6.0 & 4.2 & 0 & 1 & 1 & \\
\hline UT12-066 & $\mathrm{L}$ & & 33.9 & 2 & $\begin{array}{l}\mathrm{p} 1-2 \\
\text { and }-\end{array}$ & 1 & 2 & 2 & 17.7 & 11.6 & 2 & 2 & 0 & Major fragmented posterior end. \\
\hline UT12-066 & $\mathrm{R}$ & & 33.9 & 1 & $\begin{array}{l}\mathrm{p} 1-2 \\
\text { and }-\end{array}$ & 1 & 2 & 1 & 17.2 & 10.3 & 1 & 2 & 0 & Minor fragmented posterior end. \\
\hline UT12-067 & $\mathrm{L}$ & 76.2 & 38.3 & 0 & $\mathrm{p} 1$ and - & 1 & 2 & 2 & 23.2 & 15.0 & 1 & 2 & 1 & Some organic sheet inside the valves. \\
\hline UT12-067 & $\mathrm{R}$ & 76.2 & 38.3 & 2 & p1 and - & 1 & 2 & 2 & 21.5 & 16.3 & 2 & 2 & 1 & $\begin{array}{l}\text { Major fragmented posterior end. } \\
\text { Hole in central part. }\end{array}$ \\
\hline UT12-068 & $\mathrm{L}$ & 69.3 & 34.5 & 0 & - & 1 & 1 & 2 & 8.1 & 6.2 & 0 & 1 & 1 & Organic sheet inside the valves. \\
\hline UT12-068 & $\mathrm{R}$ & 69.3 & 34.5 & 1 & $\mathrm{p} 0$ and - & 1 & 1 & 2 & 8.1 & 5.2 & 1 & 1 & 1 & Minor fragmented posterior end. \\
\hline UT12-069 & $\mathrm{L}$ & & 26.4 & 2 & - & 1 & 2 & 1 & 13.6 & 7.2 & 2 & 2 & 2 & Major fragmented posterior end. \\
\hline UT12-069 & $\mathrm{R}$ & & 26.4 & 1 & - & 1 & 2 & 2 & 12.5 & 8.5 & 1 & 2 & 2 & Minor fragmented posterior end. \\
\hline UT12-070 & $\mathrm{L}$ & 58.1 & 27.8 & 1 & - & 0 & 1 & 1 & 7.8 & 6.0 & 0 & 1 & 1 & Scarcely fragmented. \\
\hline UT12-070 & $\mathrm{R}$ & 58.1 & 27.8 & 0 & - & 0 & 1 & 0 & 8.3 & 5.2 & 0 & 1 & 1 & \\
\hline UT12-071 & $\mathrm{L}$ & & 25.8 & 2 & p1 and - & 1 & 2 & 1 & 10.2 & 7.2 & 2 & 2 & 2 & Major fragmented posterior end. \\
\hline UT12-071 & $\mathrm{R}$ & & 25.8 & 2 & - & 0 & 2 & 0 & 10.5 & 7.0 & 2 & 2 & 2 & Major fragmented posterior end. \\
\hline
\end{tabular}


Table 3. Statistic overview of taphonomical features in valves of Unio crassus and U. tumidus. Abbreviations: Min. - minimum; Max. - maximum; Std. dev. - standard deviation.

\begin{tabular}{|c|c|c|c|c|c|c|c|}
\hline Unio crassus, left valves & Min. & Max. & Mean & Variance & Std. dev. & Median & Skewness \\
\hline Posterior-anterior length (N 22, mm) & 33.72 & 82.87 & 58.90 & 244.03 & 15.62 & 60.18 & -0.01 \\
\hline Dorso-ventral height (N 38, mm) & 18.68 & 43.76 & 32.28 & 41.33 & 6.43 & 34.67 & -0.31 \\
\hline Fragmentation (N 38) & 0 & 2 & 0.58 & 0.41 & 0.64 & 0.50 & 0.66 \\
\hline Dissolution inner surface (N 38) & 0 & 2 & 0.79 & 0.60 & 0.78 & 1 & 0.39 \\
\hline Dissolution umbo (N 33) & 1 & 2 & 1.61 & 0.25 & 0.50 & 2 & -0.46 \\
\hline Lustre inner surface (N 38) & 0 & 2 & 1.34 & 0.45 & 0.67 & 1 & -0.53 \\
\hline Dissolution umbo lenght (N 30, mm) & 4.00 & 23.52 & 11.10 & 26.47 & 5.14 & 10.05 & 1.13 \\
\hline Dissolution umbo height (N 31, mm) & 3.13 & 15.20 & 6.95 & 11.08 & 3.33 & 6.50 & 1.14 \\
\hline Periostracum non-umbo (N 38) & 0 & 2 & 0.61 & 0.62 & 0.79 & 0 & 0.85 \\
\hline Periostracum umbo (N 33) & 1 & 2 & 1.67 & 0.23 & 0.48 & 2 & -0.74 \\
\hline Ligament (N 38) & 0 & 2 & 1.58 & 0.41 & 0.64 & 2 & -1.28 \\
\hline Unio tumidus, left valves & Min. & Max. & Mean & Variance & Std dev. & Median & Skewness \\
\hline Posterior-anterior length (N 51, mm) & 40.30 & 77.80 & 60.22 & 72.56 & 8.52 & 60.70 & -0.06 \\
\hline Dorso-ventral height $(\mathrm{N} 71, \mathrm{~mm})$ & 21.40 & 38.30 & 29.61 & 12.08 & 3.48 & 29.70 & 0.02 \\
\hline Fragmentation (N 71) & 0 & 2 & 0.73 & 0.57 & 0.76 & 1 & 0.49 \\
\hline Dissolution inner surface (N 71) & 0 & 2 & 0.56 & 0.42 & 0.65 & 0 & 0.73 \\
\hline Dissolution umbo (N 71) & 0 & 2 & 1.63 & 0.26 & 0.51 & 2 & -0.89 \\
\hline Lustre inner surface (N 71) & 0 & 2 & 1.39 & 0.47 & 0.69 & 2 & -0.70 \\
\hline Dissolution umbo lenght (N 71, mm) & 6.00 & 23.20 & 10.65 & 10.69 & 3.27 & 10.30 & 1.18 \\
\hline Dissolution umbo height (N 71, mm) & 3.90 & 15.00 & 7.49 & 5.27 & 2.29 & 7.20 & 0.95 \\
\hline Periostracum non-umbo (N 71) & 0 & 2 & 0.77 & 0.58 & 0.76 & 1 & 0.41 \\
\hline Periostracum umbo (N 71) & 1 & 2 & 1.66 & 0.23 & 0.48 & 2 & -0.70 \\
\hline Ligament (N 71) & 0 & 2 & 0.34 & 0.34 & 0.58 & 0 & 1.55 \\
\hline
\end{tabular}

ing lack of periostracum in non-umbonal areas is strongly related to fragmentation, for both species (Tab. 4). Also, fragmentation is moderately related to umbonal dissolution.

Both Unio crassus and $U$. tumidus show dissolution of their inner shell surface as mostly graded either 0 or 1 (Tabs 1, 2,3). Removal of lustre of the inner shell surface ranges from 0 to 2 in grades (Tab. 3). Lustre of grade 0 occurs only in $11 \%$ valves of Unio crassus and U. tumidus (Tabs 1,2). Grades 1 and 2 are equally common in $U$. crassus valves, while there is a skewness towards grade 2 in $U$. tumidus. Lustre, which is gentle shining light that is reflected from the inner surface, is dependent upon the decay of organic matter and the degree of dissolution. Therefore, a degree of correlation could be expected between lustre and dissolution. We find a moderately positive relationship for these two taphonomical features for both, U. crassus and U. tumidus (Tab. 4). It is notable that the grades of lustre are generally equal or higher than those of dissolution (Tabs 1,2,3) indicating that the lustre can be missing without noteworthy dissolution to be observed.

Umbonal area of the valves shows notably evidence of dissolution (Tabs 1,2). For U. crassus, the dissolution area of the umbo is characterised by mean values of 11.0 and $6.9 \mathrm{~mm}$ for the length and height of the dissolution, respectively (Tab. 3). The length of dissolution ranges between 4.0 and $23.5 \mathrm{~mm}$ (std. dev. 5.1), whereas the height is from 3.1 to $15.2 \mathrm{~mm}$ (std. dev. 3.3) (Tab. 3). Linear regression with least squares of the length and height was used to statistically model their relationship and was found to indicate a strong linear correlation (not shown).

For $U$.tumidus, the dissolution in the umbonal area is indicated with averages 10.6 and $7.5 \mathrm{~mm}$, in the length and height of the corresponding area, respectively (Tab. 3). The length ranges from 6.0 to $23.2 \mathrm{~mm}$ (std. dev. 3.3), while the height from 3.9 to $15.0 \mathrm{~mm}$ (std. dev. 2.3). The measurements of length and height appeared to show a strong linear correlation. 
Table 4. The non-parametric coefficient, Spearman's r, is given for taphonomical features scored semi-quantitively for Unio crassus and U. tumidus. The coefficients are present in the lower triangle of the table, while the two-tailed probabilities for that the columns are uncorrelated are in the upper triangle. Abbreviations: F - fragmentation; Dis - dissolution inner surface; Du - dissolution umbo; Lis - lustre inner surface; Pnu - periostracum nonumbo; $\mathrm{Pu}$ - periostracum umbo; $\mathrm{Lg}$ - ligament.

\begin{tabular}{|c|c|c|c|c|c|c|c|}
\hline \multicolumn{8}{|l|}{ Unio crassus, left valves } \\
\hline Spearman & $\mathrm{F}$ & Dis & $\mathrm{Du}$ & Lis & Pnu & $\mathrm{Pu}$ & $\operatorname{Lg}$ \\
\hline Fragmentation & & 0.10 & 0.01 & 0.13 & 0.00 & 0.07 & 0.02 \\
\hline Dissolution inner surface & 0.27 & & 0.18 & 0.25 & 0.01 & 0.52 & 0.02 \\
\hline Dissolution umbo & 0.43 & 0.24 & & 0.21 & 0.05 & 0.00 & 0.35 \\
\hline Lustre inner surface & 0.25 & 0.63 & 0.23 & & 0.04 & 0.40 & 0.02 \\
\hline Periostracum non-umbo & 0.86 & 0.39 & 0.34 & 0.33 & & 0.16 & 0.00 \\
\hline Periostracum umbo & 0.32 & 0.12 & 0.88 & 0.15 & 0.25 & & 0.44 \\
\hline Ligament & 0.38 & 0.39 & 0.17 & 0.37 & 0.50 & 0.14 & \\
\hline \multicolumn{8}{|l|}{ Unio tumidus, left valves } \\
\hline Spearman & $\mathrm{F}$ & Dis & $\mathrm{Du}$ & Lis & Pnu & $\mathrm{Pu}$ & $\mathrm{Lg}$ \\
\hline Fragmentation & & 0.12 & 0.00 & 0.10 & 0.00 & 0.00 & 0.45 \\
\hline Dissolution inner surface & 0.18 & & 0.10 & 0.01 & 0.04 & 0.05 & 0.61 \\
\hline Dissolution umbo & 0.39 & 0.20 & & 0.20 & 0.04 & 0.00 & 0.07 \\
\hline Lustre inner surface & 0.20 & 0.54 & 0.15 & & 0.05 & 0.10 & 0.03 \\
\hline Periostracum non-umbo & 0.93 & 0.25 & 0.47 & 0.23 & & 0.00 & 0.28 \\
\hline Periostracum umbo & 0.36 & 0.24 & 0.94 & 0.20 & 0.44 & & 0.10 \\
\hline Ligament & 0.09 & -0.06 & 0.22 & -0.25 & 0.13 & 0.20 & \\
\hline
\end{tabular}

Moreover, it can be shown, for both studied species, that the more intensely dissolved the umbonal areas are, the more periostracum is missing in the same area (Tab. 4). This relationship appears to suggest that extensive removal of calcareous shell material furthers the detachment of especially the umbonal periostracum. Still, taphonomical features pertaining to dissolution may be only weakly related to the conditions of the non-umbonal periostracum. The preservation of the ligament appears unrelated to and thus likely unaffected by the dissolution in umbonal areas. In U. crassus, however, the ligament may be at least moderately related to the preservation of the periostracum in non-umbonal areas, that is, more intensive loss of periostracum in these areas appears to lead to poorer preservation of the ligament, or vice versa. By contrast, we find no similar indications for such a relationship in the case of $U$. tumidus.

\section{Taphonomical Indices}

The taphonomical indices are summarised in Tables 5 and 6 and their correlation coefficients are further provided in Table 7 . The overall preservation, represented by the taphoSUM and perioSUM indices, indicates no relationships with the dorso-ventral height (Tab. 7). However, the dorso-ventral height and dissUMBO-index are moderately related, at least in $U$. crassus. This relationship is weaker for $U$.tumidus. The dissUMBO-index is weakly and moderately related to the perioSUM-index, in $U$. crassus and U. tumidus, respectively. Both species show a strong relationship between the perioSUM and taphoSUM indices, which may not be surprising, however, as they share some of the same input parameters.

\section{Radiocarbon dates}

The ventral margin of seven shells of Unio crassus were exposed to ${ }^{14} \mathrm{C}$ dating (Tab. 8). Of these, five shells represent modern ${ }^{14} \mathrm{C}$ ages, their post-1950 cal. AD ages ranging from 1956 to $2001 \mathrm{cal}$. AD. Two shells show ages of 1842 and $1848 \mathrm{cal}$. AD, with relatively wide calibrated radiocarbon age distributions.

\section{Discussion}

Our finds highlight that the possibility of time-averaging of $U$. crassus shells exists in the death assemblages 
Figure 4. Ternary taphograms for taphonomical features scored semi-quantitatively for the left valves of Unio crassus and $U$. tumidus. Labels of the data points for $U$. crassus refer to various sites, while all of those for U. tumidus represent site 12 .
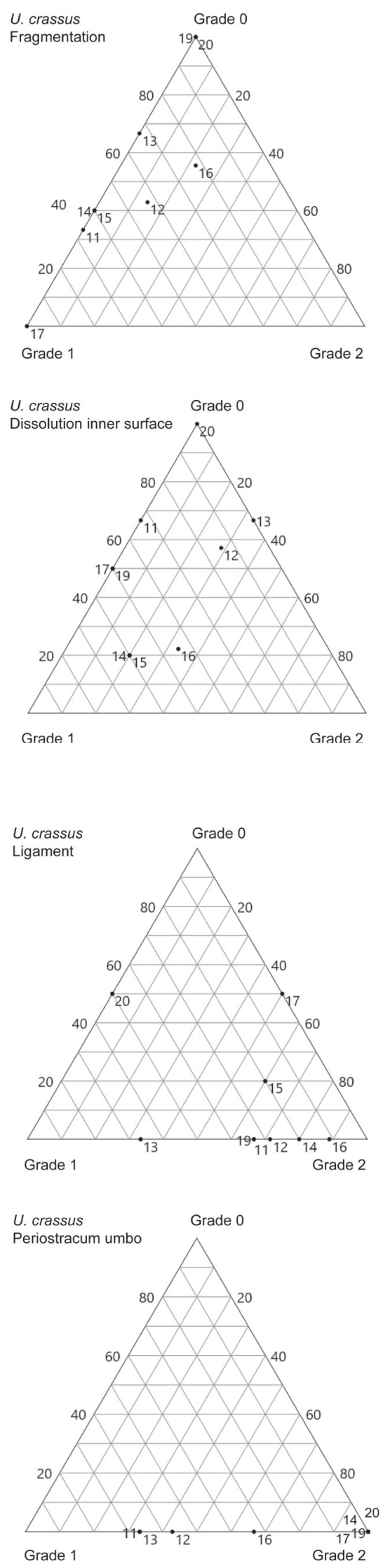
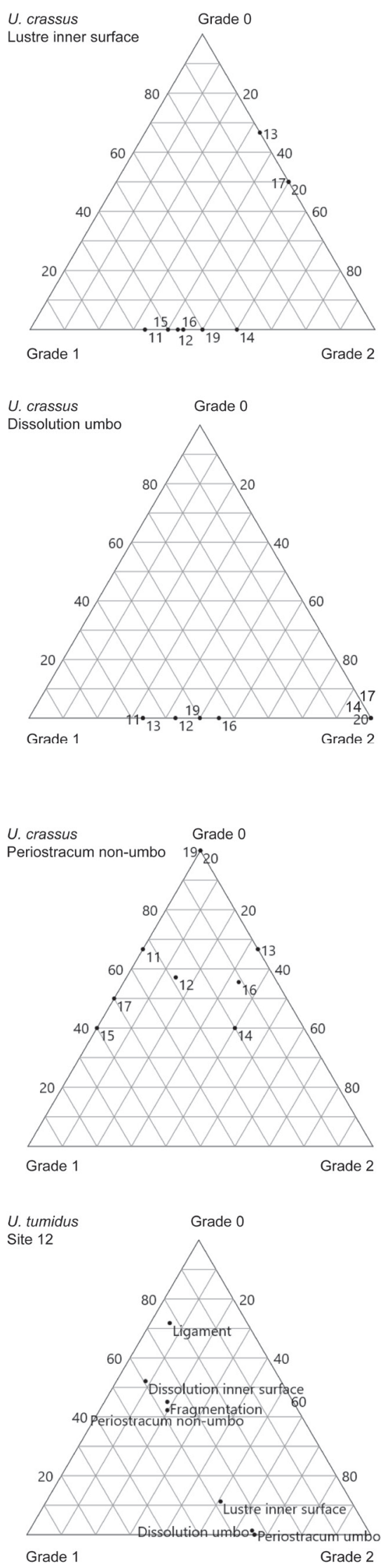
obtained from the bottom sediment of river Vantaanjoki. Moreover, preservational status of the individual valves was investigated and the value of the method with taphonomical indices illustrated, to further demonstrate the benefits of combining the ${ }^{14} \mathrm{C}$ and taphonomy approaches. These results are discussed below in more details, with potential limitations of our findings.

\section{Radiocarbon dates and time-averaging}

The shells were assumed, as a premise, to be modern, i.e. their ${ }^{14} \mathrm{C}$ ages to represent the post-1950 AD ages. The ${ }^{14} \mathrm{C}$ dates confirmed this assumption for at least five of Unio crassus shells (Tab. 8). However, two of the shells were noticeably older, not modern in age, i.e. resulting in

Table 5. Taphonomical indices for Unio crassus. Abbreviations: $\mathrm{V}$ - valve ( $\mathrm{L}=$ left; $\mathrm{R}=$ right); $\mathrm{H}$ - dorso-ventral height; $\mathrm{dUMBO}$ - dissUMBO-index; tSUM - taphoSUM-index; pSUM - perioSUM-index.

\begin{tabular}{|c|c|c|c|c|c|}
\hline Shell No. & V & $\mathrm{H}(\mathrm{mm})$ & dUMBO & tSUM & pSUM \\
\hline UC11-001 & $\mathrm{L}$ & 25.9 & 12.88 & 8 & 2 \\
\hline UC11-001 & $\mathrm{R}$ & 25.9 & & 6 & 1 \\
\hline UC11-002 & $\mathrm{L}$ & 30.9 & 70.25 & 7 & 2 \\
\hline UC11-002 & $\mathrm{R}$ & 30.9 & & 2 & 0 \\
\hline UC11-003 & $\mathrm{L}$ & 34.4 & 20.74 & 6 & 1 \\
\hline UC11-003 & $\mathrm{R}$ & 34.4 & & 5 & 1 \\
\hline UC12-001 & $\mathrm{L}$ & 24.2 & 10.96 & 4 & 1 \\
\hline UC12-001 & $\mathrm{R}$ & 24.2 & & 4 & 1 \\
\hline UC12-002 & $\mathrm{L}$ & 30.6 & 52.54 & 9 & 3 \\
\hline UC12-002 & $\mathrm{R}$ & 30.6 & & 11 & 3 \\
\hline UC12-003 & $\mathrm{L}$ & & & & \\
\hline UC12-003 & $\mathrm{R}$ & 34.5 & & 11 & 3 \\
\hline UC12-004 & $\mathrm{L}$ & 32.4 & 30.74 & 7 & 2 \\
\hline UC12-004 & $\mathrm{R}$ & & & 9 & 3 \\
\hline UC12-005 & $\mathrm{L}$ & 35.5 & 28.22 & 8 & 1 \\
\hline UC12-005 & $\mathrm{R}$ & 35.5 & & 8 & 1 \\
\hline UC12-006 & $\mathrm{L}$ & 36.2 & 76.33 & 10 & 2 \\
\hline UC12-006 & $\mathrm{R}$ & 36.2 & & 10 & 2 \\
\hline UC12-007 & $\mathrm{L}$ & & & 14 & 4 \\
\hline UC12-007 & $\mathrm{R}$ & 41.3 & & 13 & 4 \\
\hline UC12-008 & $\mathrm{L}$ & 37.6 & 52.22 & 4 & 1 \\
\hline UC12-008 & $\mathrm{R}$ & 37.6 & & 6 & 1 \\
\hline UC13-001 & $\mathrm{L}$ & 25.1 & 17.74 & 3 & 1 \\
\hline UC13-001 & $\mathrm{R}$ & 25.1 & & 4 & 1 \\
\hline UC13-002 & $\mathrm{L}$ & 33.6 & & 13 & 4 \\
\hline UC13-002 & $\mathrm{R}$ & 33.6 & & 14 & 4 \\
\hline UC13-003 & $\mathrm{L}$ & 35.0 & & 3 & 1 \\
\hline UC13-003 & $\mathrm{R}$ & 35.0 & & 3 & 1 \\
\hline UC14-001 & $\mathrm{L}$ & 18.7 & 21.77 & 8 & 2 \\
\hline UC14-001 & $\mathrm{R}$ & 18.7 & & 4 & 0 \\
\hline UC14-002 & $\mathrm{L}$ & 24.2 & 20.51 & 9 & 3 \\
\hline UC14-002 & $\mathrm{R}$ & 24.2 & & 9 & 2 \\
\hline UC14-003 & $\mathrm{L}$ & 27.2 & 72.82 & 8 & 2 \\
\hline UC14-003 & $\mathrm{R}$ & 27.2 & & 9 & 2 \\
\hline UC14-004 & $\mathrm{L}$ & 35.5 & 242.91 & 13 & 4 \\
\hline UC14-004 & $\mathrm{R}$ & 35.5 & & 14 & 4 \\
\hline UC14-005 & $\mathrm{L}$ & 37.2 & 261.67 & 12 & 4 \\
\hline UC14-005 & $\mathrm{R}$ & 37.2 & & 13 & 4 \\
\hline UC15-002 & $\mathrm{L}$ & 35.9 & & 5 & 1 \\
\hline
\end{tabular}

\begin{tabular}{|c|c|c|c|c|c|}
\hline Shell No. & V & $\mathrm{H}(\mathrm{mm})$ & dUMBO & tSUM & pSUM \\
\hline UC15-002 & $\mathrm{R}$ & 35.9 & & 5 & 1 \\
\hline UC15-001 & $\mathrm{L}$ & 31.7 & & 7 & 1 \\
\hline UC15-001 & $\mathrm{R}$ & 31.7 & & 8 & 2 \\
\hline UC15-003 & $\mathrm{L}$ & 36.2 & & 7 & 1 \\
\hline UC15-003 & $\mathrm{R}$ & 36.2 & & 9 & 2 \\
\hline UC15-004 & $\mathrm{L}$ & 35.0 & & 4 & 0 \\
\hline UC15-004 & $\mathrm{R}$ & 35.0 & & 5 & 1 \\
\hline UC15-005 & $\mathrm{L}$ & 42.2 & & 2 & 0 \\
\hline UC15-005 & $\mathrm{R}$ & 42.2 & & 2 & 0 \\
\hline UC16-001 & $\mathrm{L}$ & 22.6 & 34.06 & 13 & 4 \\
\hline UC16-001 & $\mathrm{R}$ & 22.6 & & 13 & 4 \\
\hline UC16-002 & $\mathrm{L}$ & 25.5 & 46.85 & 14 & 4 \\
\hline UC16-002 & $\mathrm{R}$ & 25.5 & & 12 & 4 \\
\hline UC16-003 & $\mathrm{L}$ & 23.8 & 25.94 & 7 & 2 \\
\hline UC16-003 & $\mathrm{R}$ & 23.8 & & 7 & 2 \\
\hline UC16-004 & $\mathrm{L}$ & 28.7 & 25.02 & 8 & 2 \\
\hline UC16-004 & $\mathrm{R}$ & 28.7 & & 8 & 2 \\
\hline UC16-005 & $\mathrm{L}$ & 27.6 & 40.93 & 6 & 2 \\
\hline UC16-005 & $\mathrm{R}$ & 27.6 & & 3 & 0 \\
\hline UC16-006 & $\mathrm{L}$ & 37.4 & 95.31 & 11 & 4 \\
\hline UC16-006 & $\mathrm{R}$ & 37.4 & & 12 & 4 \\
\hline UC16-007 & $\mathrm{L}$ & 35.3 & 65.96 & 6 & 1 \\
\hline UC16-007 & $\mathrm{R}$ & 35.3 & & 9 & 2 \\
\hline UC16-008 & $\mathrm{L}$ & 36.3 & 54.84 & 8 & 1 \\
\hline UC16-008 & $\mathrm{R}$ & 36.3 & & 8 & 1 \\
\hline UC16-009 & $\mathrm{L}$ & 37.1 & 90.75 & 9 & 2 \\
\hline UC16-009 & $\mathrm{R}$ & 37.1 & & 7 & 2 \\
\hline UC17-001 & $\mathrm{L}$ & 36.3 & 205.35 & 5 & 2 \\
\hline UC17-001 & $\mathrm{R}$ & 36.3 & 167.73 & 4 & 2 \\
\hline UC17-002 & $\mathrm{L}$ & 42.6 & 231.69 & 11 & 3 \\
\hline UC17-002 & $\mathrm{R}$ & 42.6 & 183.40 & 8 & 2 \\
\hline UC19-001 & $\mathrm{L}$ & 20.0 & 23.36 & 5 & 2 \\
\hline UC19-001 & $\mathrm{R}$ & 20.0 & & 5 & 2 \\
\hline UC19-002 & $\mathrm{L}$ & 26.8 & 31.35 & 9 & 2 \\
\hline UC19-002 & $\mathrm{R}$ & 26.8 & & 10 & 2 \\
\hline UC20-001 & $\mathrm{L}$ & 43.8 & 85.01 & 4 & 2 \\
\hline UC20-001 & $\mathrm{R}$ & 43.8 & 132.04 & 4 & 2 \\
\hline UC20-002 & $\mathrm{L}$ & 36.3 & 88.18 & 7 & 2 \\
\hline UC20-002 & $\mathrm{R}$ & 36.3 & 78.96 & 8 & 2 \\
\hline
\end{tabular}


Table 6. Taphonomical indices for Unio tumidus. Abbreviations: $\mathrm{V}$ - valve $(\mathrm{L}=$ left; $\mathrm{R}=$ right $)$; $\mathrm{H}$ - dorso-ventral height; $\mathrm{dUMBO}$ - dissUMBO-index; tSUM - taphoSUM-index; pSUM - perioSUM-index.

\begin{tabular}{|c|c|c|c|c|c|}
\hline Shell No. & V & $\mathrm{H}(\mathrm{mm})$ & dUMBO & tSUM & pSUM \\
\hline UT12-001 & $\mathrm{L}$ & 28.8 & 32.73 & 7 & 3 \\
\hline UT12-001 & $\mathrm{R}$ & 28.8 & 38.52 & 3 & 1 \\
\hline UT12-002 & $\mathrm{L}$ & 30.0 & 31.40 & 5 & 2 \\
\hline UT12-002 & $\mathrm{R}$ & 30.0 & 19.68 & 7 & 2 \\
\hline UT12-003 & $\mathrm{L}$ & 29.5 & 64.65 & 5 & 2 \\
\hline UT12-003 & $\mathrm{R}$ & 29.5 & 76.11 & 6 & 2 \\
\hline UT12-004 & $\mathrm{L}$ & 27.1 & 51.77 & 7 & 3 \\
\hline UT12-004 & $\mathrm{R}$ & 27.1 & 55.21 & 9 & 4 \\
\hline UT12-005 & $\mathrm{L}$ & 27.5 & 65.14 & 12 & 4 \\
\hline UT12-005 & $\mathrm{R}$ & 27.5 & 44.21 & 9 & 3 \\
\hline UT12-006 & $\mathrm{L}$ & 37.0 & 161.21 & 12 & 4 \\
\hline UT12-006 & $\mathrm{R}$ & 37.0 & 156.38 & 12 & 4 \\
\hline UT12-007 & $\mathrm{L}$ & 29.2 & 43.50 & 3 & 1 \\
\hline UT12-007 & $\mathrm{R}$ & 29.2 & 57.82 & 5 & 1 \\
\hline UT12-008 & $\mathrm{L}$ & 30.0 & 31.42 & 4 & 1 \\
\hline UT12-008 & $\mathrm{R}$ & 30.0 & 24.23 & 4 & 1 \\
\hline UT12-009 & $\mathrm{L}$ & 26.1 & 53.43 & 8 & 3 \\
\hline UT12-009 & $\mathrm{R}$ & 26.1 & 50.34 & 7 & 2 \\
\hline UT12-010 & $\mathrm{L}$ & 23.0 & 53.11 & 4 & 2 \\
\hline UT12-010 & $\mathrm{R}$ & 23.0 & 55.57 & 4 & 2 \\
\hline UT12-011 & $\mathrm{L}$ & 32.4 & 83.11 & 8 & 3 \\
\hline UT12-011 & $\mathrm{R}$ & 32.4 & 130.93 & 8 & 3 \\
\hline UT12-012 & $\mathrm{L}$ & 31.3 & 19.24 & 4 & 1 \\
\hline UT12-012 & $\mathrm{R}$ & 31.3 & 18.29 & 4 & 1 \\
\hline UT12-013 & $\mathrm{L}$ & 26.8 & 35.03 & 2 & 1 \\
\hline UT12-013 & $\mathrm{R}$ & 26.8 & 38.23 & 4 & 2 \\
\hline UT12-014 & $\mathrm{L}$ & 28.3 & 34.95 & 6 & 2 \\
\hline UT12-014 & $\mathrm{R}$ & 28.3 & 40.45 & 6 & 2 \\
\hline UT12-015 & $\mathrm{L}$ & 29.4 & 107.40 & 9 & 3 \\
\hline UT12-015 & $\mathrm{R}$ & 29.4 & 102.86 & 8 & 3 \\
\hline UT12-016 & $\mathrm{L}$ & 26.0 & 36.16 & 7 & 3 \\
\hline UT12-016 & $\mathrm{R}$ & 26.0 & 38.69 & 4 & 2 \\
\hline UT12-017 & $\mathrm{L}$ & 31.3 & 36.76 & 6 & 3 \\
\hline UT12-017 & $\mathrm{R}$ & 31.3 & 36.74 & 6 & 3 \\
\hline UT12-018 & $\mathrm{L}$ & 27.7 & 44.32 & 5 & 2 \\
\hline UT12-018 & $\mathrm{R}$ & 27.7 & 54.81 & 4 & 2 \\
\hline UT12-019 & $\mathrm{L}$ & 35.3 & 119.90 & 8 & 3 \\
\hline UT12-019 & $\mathrm{R}$ & 35.3 & 166.72 & 6 & 2 \\
\hline UT12-020 & $\mathrm{L}$ & 21.4 & 64.21 & 7 & 2 \\
\hline UT12-020 & $\mathrm{R}$ & 21.4 & 80.35 & 9 & 3 \\
\hline UT12-021 & $\mathrm{L}$ & 32.0 & 25.40 & 6 & 1 \\
\hline UT12-021 & $\mathrm{R}$ & 32.0 & 29.82 & 6 & 1 \\
\hline UT12-022 & $\mathrm{L}$ & 35.4 & 100.84 & 9 & 3 \\
\hline UT12-022 & $\mathrm{R}$ & 35.4 & 64.15 & 7 & 2 \\
\hline UT12-023 & $\mathrm{L}$ & 25.9 & 40.17 & 9 & 3 \\
\hline UT12-023 & $\mathrm{R}$ & 25.9 & 34.71 & 7 & 2 \\
\hline UT12-024 & $\mathrm{L}$ & 33.3 & 32.76 & 2 & 1 \\
\hline UT12-024 & $\mathrm{R}$ & 33.3 & 26.12 & 4 & 1 \\
\hline
\end{tabular}

\begin{tabular}{|c|c|c|c|c|c|}
\hline Shell No. & V & $\mathrm{H}(\mathrm{mm})$ & dUMBO & tSUM & pSUM \\
\hline UT12-025 & $\mathrm{L}$ & 31.7 & 56.2 & 2 & 1 \\
\hline UT12-025 & $\mathrm{R}$ & 31.7 & 35.37 & 2 & 1 \\
\hline UT12-026 & $\mathrm{L}$ & 30.0 & 65.78 & 10 & 4 \\
\hline UT12-026 & $\mathrm{R}$ & 30.0 & 82.36 & 6 & 2 \\
\hline UT12-027 & $\mathrm{L}$ & 28.2 & 104.51 & 6 & 2 \\
\hline UT12-027 & $\mathrm{R}$ & 28.2 & 143.27 & 8 & 3 \\
\hline UT12-028 & $\mathrm{L}$ & 28.6 & 29.14 & 4 & 1 \\
\hline UT12-028 & $\mathrm{R}$ & 28.6 & 34.74 & 4 & 1 \\
\hline UT12-029 & $\mathrm{L}$ & 33.4 & 95.24 & 11 & 4 \\
\hline UT12-029 & $\mathrm{R}$ & 33.4 & 105.70 & 8 & 3 \\
\hline UT12-030 & $\mathrm{L}$ & 26.6 & 42.47 & 6 & 2 \\
\hline UT12-030 & $\mathrm{R}$ & 26.6 & 45.26 & 6 & 2 \\
\hline UT12-031 & $\mathrm{L}$ & 31.1 & 57.25 & 10 & 3 \\
\hline UT12-031 & $\mathrm{R}$ & 31.1 & 103.85 & 10 & 3 \\
\hline UT12-032 & $\mathrm{L}$ & 31.2 & 55.30 & 10 & 3 \\
\hline UT12-032 & $\mathrm{R}$ & 31.2 & 61.15 & 10 & 3 \\
\hline UT12-033 & $\mathrm{L}$ & 32.3 & 34.15 & 5 & 2 \\
\hline UT12-033 & $\mathrm{R}$ & 32.3 & 32.89 & 5 & 2 \\
\hline UT12-034 & $\mathrm{L}$ & 25.4 & 62.79 & 6 & 2 \\
\hline UT12-034 & $\mathrm{R}$ & 25.4 & 80.83 & 6 & 2 \\
\hline UT12-035 & $\mathrm{L}$ & 30.3 & 61.00 & 9 & 3 \\
\hline UT12-035 & $\mathrm{R}$ & 30.3 & 63.59 & 9 & 3 \\
\hline UT12-036 & $\mathrm{L}$ & 35.1 & 123.07 & 6 & 2 \\
\hline UT12-036 & $\mathrm{R}$ & 35.1 & 206.01 & 8 & 3 \\
\hline UT12-037 & $\mathrm{L}$ & 26.8 & 31.79 & 9 & 3 \\
\hline UT12-037 & $\mathrm{R}$ & 26.8 & 42.43 & 10 & 4 \\
\hline UT12-038 & $\mathrm{L}$ & 32.9 & 190.27 & 10 & 3 \\
\hline UT12-038 & $\mathrm{R}$ & 32.9 & 107.55 & 10 & 3 \\
\hline UT12-039 & $\mathrm{L}$ & 28.7 & 32.90 & 3 & 1 \\
\hline UT12-039 & $\mathrm{R}$ & 28.7 & 33.18 & 5 & 2 \\
\hline UT12-040 & $\mathrm{L}$ & 31.7 & 78.96 & 7 & 3 \\
\hline UT12-040 & $\mathrm{R}$ & 31.7 & 94.26 & 9 & 4 \\
\hline UT12-041 & $\mathrm{L}$ & 33.6 & 68.55 & 9 & 3 \\
\hline UT12-041 & $\mathrm{R}$ & 33.6 & 76.89 & 9 & 3 \\
\hline UT12-042 & $\mathrm{L}$ & 29.8 & 33.15 & 4 & 1 \\
\hline UT12-042 & $\mathrm{R}$ & 29.8 & 26.07 & 6 & 2 \\
\hline UT12-043 & $\mathrm{L}$ & 30.3 & 54.91 & 7 & 2 \\
\hline UT12-043 & $\mathrm{R}$ & 30.3 & 62.02 & 5 & 1 \\
\hline UT12-044 & $\mathrm{L}$ & 21.9 & 65.63 & 7 & 2 \\
\hline UT12-044 & $\mathrm{R}$ & 21.9 & 53.15 & 8 & 2 \\
\hline UT12-045 & $\mathrm{L}$ & 32.3 & 117.42 & 11 & 4 \\
\hline UT12-045 & $\mathrm{R}$ & 32.3 & 90.26 & 9 & 3 \\
\hline UT12-046 & $\mathrm{L}$ & 28.4 & 108.80 & 8 & 3 \\
\hline UT12-046 & $\mathrm{R}$ & 28.4 & 87.00 & 7 & 3 \\
\hline UT12-047 & $\mathrm{L}$ & 33.2 & 113.44 & 5 & 2 \\
\hline UT12-047 & $\mathrm{R}$ & 33.2 & 79.71 & 5 & 2 \\
\hline UT12-048 & $\mathrm{L}$ & 28.9 & 84.88 & 12 & 4 \\
\hline UT12-048 & $\mathrm{R}$ & 28.9 & 155.99 & 10 & 3 \\
\hline
\end{tabular}


Table 6. Continued.

\begin{tabular}{lccccc}
\hline Shel1 No. & V & H (mm) & dUMBO & tSUM & pSUM \\
\hline UT12-049 & L & 29.1 & 53.44 & 4 & 1 \\
UT12-049 & R & 29.1 & 86.76 & 6 & 2 \\
UT12-050 & L & 30.0 & 29.84 & 3 & 1 \\
UT12-050 & R & 30.0 & 27.19 & 3 & 1 \\
UT12-051 & L & 27.3 & 48.33 & 8 & 3 \\
UT12-051 & R & 27.3 & 47.70 & 8 & 3 \\
UT12-052 & L & 23.1 & 27.99 & 6 & 1 \\
UT12-052 & R & 23.1 & 23.58 & 5 & 1 \\
UT12-053 & L & 29.7 & 67.95 & 8 & 2 \\
UT12-053 & R & 29.7 & 97.23 & 8 & 2 \\
UT12-054 & L & 27.0 & 45.98 & 8 & 3 \\
UT12-054 & R & 27.0 & 61.02 & 8 & 3 \\
UT12-055 & L & 33.2 & 68.11 & 8 & 3 \\
UT12-055 & R & 33.2 & 84.25 & 10 & 3 \\
UT12-056 & L & 26.1 & 39.67 & 7 & 3 \\
UT12-056 & R & 26.1 & 43.79 & 7 & 2 \\
UT12-057 & L & 24.0 & 39.34 & 8 & 2 \\
UT12-057 & R & 24.0 & 32.57 & 8 & 2 \\
UT12-058 & L & 31.3 & 94.85 & 9 & 3 \\
UT12-058 & R & 31.3 & 88.83 & 11 & 4 \\
UT12-059 & L & 27.1 & 93.19 & 11 & 4 \\
UT12-059 & R & 27.1 & 73.44 & 11 & 4 \\
UT12-060 & L & 29.9 & 90.36 & 7 & 3 \\
\hline
\end{tabular}

\begin{tabular}{lccccc}
\hline Shell No. & V & H (mm) & dUMBO & tSUM & pSUM \\
\hline UT12-060 & R & 29.9 & 86.14 & 7 & 3 \\
UT12-061 & L & 30.8 & 137.01 & 11 & 4 \\
UT12-061 & R & 30.8 & 106.99 & 9 & 3 \\
UT12-062 & L & 29.9 & 21.94 & 3 & 1 \\
UT12-062 & R & 29.9 & 51.49 & 9 & 4 \\
UT12-063 & L & 34.7 & 48.66 & 4 & 1 \\
UT12-063 & R & 34.7 & 35.63 & 4 & 1 \\
UT12-064 & L & 28.4 & 90.30 & 9 & 4 \\
UT12-064 & R & 28.4 & 86.76 & 8 & 4 \\
UT12-065 & L & 26.8 & 22.61 & 3 & 1 \\
UT12-065 & R & 26.8 & 19.54 & 3 & 1 \\
UT12-066 & L & 33.9 & 161.34 & 11 & 4 \\
UT12-066 & R & 33.9 & 139.11 & 8 & 3 \\
UT12-067 & L & 38.3 & 272.40 & 9 & 3 \\
UT12-067 & R & 38.3 & 275.23 & 12 & 4 \\
UT12-068 & L & 34.5 & 39.64 & 6 & 1 \\
UT12-068 & R & 34.5 & 33.46 & 8 & 2 \\
UT12-069 & L & 26.4 & 77.12 & 12 & 4 \\
UT12-069 & R & 26.4 & 83.38 & 11 & 3 \\
UT12-070 & L & 27.8 & 36.77 & 5 & 1 \\
UT12-070 & R & 27.8 & 33.80 & 3 & 1 \\
UT12-071 & L & 25.8 & 57.63 & 12 & 4 \\
UT12-071 & R & 25.8 & 57.53 & 10 & 4 \\
\hline
\end{tabular}

pre-1950 ages after the calibration. It is thus possible that some of the river mussels did not live contemporaneous with each other in their habitat, considering that life span of $U$. crassus is about 20-30 years (Welter Schultes 2013),

Table 7. Spearman correlation coefficients for taphonomical indices from left valves of Unio crassus and U. tumidus. Abbreviations: $\mathrm{H}$ - dorso-ventral height; dUMBO - dissUMBO-index; tSUM taphoSUM-index; pSUM - perioSUM-index.

\begin{tabular}{|c|c|c|c|c|}
\hline \multicolumn{5}{|c|}{ Unio crassus, left valves } \\
\hline Spearman & $\mathrm{H}$ & dUMBO & tSUM & pSUM \\
\hline Height & & 0.02 & 0.66 & 0.41 \\
\hline dissUMBO-index & 0.75 & & 0.04 & 0.02 \\
\hline taphoSUM-index & -0.07 & 0.37 & & 0.00 \\
\hline perioSUM-index & -0.14 & 0.43 & 0.82 & \\
\hline \multicolumn{5}{|c|}{ Unio tumidus, left valves } \\
\hline Spearman & $\mathrm{H}$ & dUMBO & tSUM & pSUM \\
\hline Height & & 0.74 & 0.23 & 0.40 \\
\hline dissUMBO-index & 0.33 & & 0.00 & 0.00 \\
\hline taphoSUM-index & 0.10 & 0.63 & & 0.00 \\
\hline perioSUM-index & 0.07 & 0.64 & 0.89 & \\
\hline
\end{tabular}

an estimate of longevity confirmed, generally, also for $U$. crassus inhabiting the River Vantaa (Helama et al. 2017). Moreover, the range of calibrated ${ }^{14} \mathrm{C}$ ages indicates that time-averaging of $U$. crassus shells exists in the death assemblages, due to sedimentological or biological processes of mixing. Similarities in life position (i.e. the position in which the mussels live buried in the river bottom with the posterior end often above the river bottom (e.g. Mandahl-Barth 1949, Ellis 1978) and environmental observations near the sites (Vahtera et al. 2010) suggest that shells of $U$. tumidus also may be time-averaged. The phenomenon of time-averaging (i.e. temporal dissociation of the fossils found within a single stratum) is well known and has particularly been recognized in the marine fossil record (e.g. Fürsich \& Aberhan 1990, Kidwell \& Bosence 1991, Flessa et al. 1993, Kidwell 1998, Nielsen et al. 2018). Time-averaging has also been documented from death assemblages of freshwater molluscs, even at a multi-millennial scale (e.g. Kotzian \& Simões 2006, Kusnerik et al. 2020). The fidelity of fossil assemblages, death assemblages and local living communities has been investigated from fluvial and lacustrine settings, for example, similarities in taxonomic composition and relative abundance of species (e.g. Pip 1988; Briggs et al. 1990; Martello et al. 2006; Erthal et al. 2011, 2015; Tietze 
Table 8. Radiocarbon dates from the ventral shell margin of Unio crassus. Abbreviations: BP - before present; calAD - calendar age; pMC percentage of modern carbon.

\begin{tabular}{|c|c|c|c|c|c|c|}
\hline Sample name & Lab. no. & Age ${ }^{14} \mathrm{C}( \pm 1 \sigma)$ & Remarks & $1 \sigma$ probability (calAD) & $2 \sigma$ probability (calAD) & Median (calAD) \\
\hline \multirow[t]{2}{*}{ FMNH-UC12-007-V } & Poz-37124 & $108.44 \pm 0.36 \mathrm{pMC}$ & Modern & $2001(68.2 \%) 2002$ & $1957(4.0 \%) 1957$ & 2001 \\
\hline & & & & & $2000(91.4 \%) 2003$ & \\
\hline \multirow[t]{2}{*}{ FMNH-UC13-002-V } & Poz-37125 & $137.14 \pm 0.43 \mathrm{pMC}$ & Modern & $1974(68.2 \%) 1976$ & $1962(5.6 \%) 1962$ & 1975 \\
\hline & & & & & $1974(89.8 \%) 1976$ & \\
\hline FMNH-UC15-003-V & Poz-37127 & $101.42 \pm 0.34 \mathrm{pMC}$ & Modern & $1955(68.2 \%) 1956$ & $1955(95.4 \%) 1956$ & 1956 \\
\hline \multirow[t]{3}{*}{ FMNH-UC13-001-V } & Poz-37128 & $75 \pm 30 \mathrm{BP}$ & & $1697(20.1 \%) 1725$ & $1690(24.6 \%) 1730$ & 1848 \\
\hline & & & & $1815(14.2 \%) 1835$ & $1810(70.8 \%) 1925$ & \\
\hline & & & & $1878(33.8 \%) 1917$ & & \\
\hline \multirow[t]{2}{*}{ FMNH-UC17-001-V } & Poz-37129 & $102.98 \pm 0.35 \mathrm{pMC}$ & Modern & $1955(9.7 \%) 1955$ & 1955 (95.4\%) 1957 & 1956 \\
\hline & & & & $1956(58.5 \%) 1956$ & & \\
\hline FMNH-UC20-001-V & Poz-37130 & $102.61 \pm 0.34 \mathrm{pMC}$ & Modern & $1955(68.2 \%) 1956$ & $1955(95.4 \%) 1957$ & 1956 \\
\hline \multirow[t]{3}{*}{ FMNH-UC13-003-V } & Poz-37131 & $90 \pm 30 \mathrm{BP}$ & & $1697(22.0 \%) 1726$ & $1685(26.3 \%) 1733$ & 1842 \\
\hline & & & & $1815(15.7 \%) 1836$ & $1807(69.1 \%) 1928$ & \\
\hline & & & & $1877(30.5 \%) 1917$ & & \\
\hline
\end{tabular}

\& De Francesco 2012). As a spectacular example from the Albian Bear River Formation (Wyoming), autochthonous freshwater and brackish water molluscan remains can co-occur in the same beds, signifying environmental condensation related to time-averaging of salinity levels and benthic communities (Fürsich \& Kauffman 1984). In the case of our depositional setting, as expected from the shoreline displacement and land emergence from the sea of the present-day Vantaa river basin (Tikkanen 1989, Tikkanen \& Ruth 2003), there should be no such dramatic changes expected to be found in the investigated death assemblages. The ${ }^{14} \mathrm{C}$ dates point towards temporal resolution of the death assemblages being the last 1-2 centuries at maximum. The actual time span of timeaveraging, however, might extend further back in time, as our sample may not have been large enough to detect the oldest (infrequent) specimens. Moreover, a distinction between the non-modern and modern empty shells from the River Vantaa cannot be based upon their taphonomical features. For example, the non-modern shells (UC13-001, UC13-003) have eminently preserved lustre in the inner shell surface, despite of their longer term exposure to post-mortem taphonomical processes. Such shells may have been buried below the taphonomically active zone (i.e. where taphonomical loss particularly occurs) and re-entered it at a later point (e.g. Davies et al. 1989; Olszewski 1999, 2004). These implications concur with the general standpoint of shell preservation observed in shallow-marine habitats indicating that the preservational status may be an untrustworthy indicator of time after death (Flessa et al. 1993). This is how the freshwater shells from our study appear to reinforce the corresponding taphonomical viewpoint, obtained previously for shells from marine depositional settings. Finally, the possibility that the unexpectedly old ${ }^{14} \mathrm{C}$ ages of the two shells results from particles eroded from deposits older than the shells should be borne in mind. Such an interpretation could be supported by ${ }^{14} \mathrm{C}$ ages of $805-1135 \mathrm{BP}$ (uncalibrated) as obtained for particulate organic carbon in some Finnish streams, suggested to originate from deeper within the peat profile, possibly associated with reworked bank or stream sediments (Billett et al. 2012). The area of the Vantaanjoki basin contains peatlands, including the drained mire of Silwäkeneva (Tikkanen 1989) located upstream to these sites. As a caveat, it is difficult to disentangle why only a smaller portion of the shells would be affected by such old food effect. We note that to exclude this hypothesis, an intensive campaign for determining ${ }^{14} \mathrm{C}$ of food particle available and suitable for unionids should be conducted in the same river. Such an investigation is beyond the scope of this taphonomical study.

\section{Preservation}

The preservational status of the individual valves was assumed to be represented by the taphoSUM-index. The index is a sum of taphonomical grades, its minimum level at the value 2 (Tabs 5,6), meaning that every valve had at least some alteration from taphonomical processes. If the calculation of taphoSUM-index is unbiased, then we could conclude that the general preservation is independent of valve size. This is indeed suggested by negligible correlations between dorso-ventral height of the shells and the taphoSUM-index. Similar findings were evident also for the perioSUM-index. However, the umbonal dis- 
solution, quantified here by the dissUMBO-index, appears to depend, to an extent, on the dorso-ventral height of the shell (Tab. 7), which in turn approximates the biological age of the mussel. That the umbonal dissolution had continued through the post-mortem decay processes is indicated by the positive relationship between the dissUMBO and perioSUM indices, the latter remaining unrelated to the dorso-ventral height of the shells (Tab. 7). Umbonal corrosion (i.e. attrition by chemical process) is a frequent phenomenon in living Unio specimens (e.g. MandahlBarth \& Bondesen 1949). The phenomenon appears also in empty shells of $U$. crassus and $U$. tumidus in museum collections (Knudsen et al. 2003). Living river mussels such as Margaritifera margaritifera (Linnaeus, 1758) and $U$. crassus tend to get more severely corroded umbones as their ontogenetic age progresses (e.g. Helama \& Valovirta 2007). The corrosion, which commonly occurs in unionid shells, is caused by river water that is unsaturated in aragonite (Cummins 1994) which, albeit $\mathrm{pH}$ of the water in River Vantaa is close to neutral, is a likely factor also behind the observed patterns of corrosion in the studied shells. Besides the sampling strategy, the unsaturated water could similarly explain the absence of smaller dead shells in the material from the River Vantaa. That is, the size selectivity towards larger shells in death assemblages can happen by smaller shells being more prone to dissolution (Cummins 1994). Of post-mortem processes, both $U$. crassus and $U$. tumidus show lustre and dissolution of the inner shell surface to be correlated moderately positively. There is no indication of specific difference in post-mortem corrosion.

The ligament tends to be better preserved in U. tumidus than $U$. crassus (Tab. 3). As the ligament formed close to the umbones, the detachment of ligament might be related to corrosion. The umbonal dissolution appears weakly correlated to the ligament preservation in U. crassus, and no correlation in U. tumidus (Tab. 4), meaning that the results are inconclusive. Another explanation for ligament detachment could be that $U$. crassus prefers waters with strong river currents, according to Mandahl-Barth (1949), and its empty shells therefore might be more exposed to transportation along the river bottom than U. tumidus. However, we do not have any evidence for differential abrasion and fragmentation confirming this.

A number of the shells were to some extent fragmented, regardless of the studied species (Tabs 1,2). The frequently observed pattern demonstrated that the shells are most often broken irregularly in their posterior end, which can be due to various reasons. Although pearl hunting using a knife would leave dorso-posterior fractures in the shells (Helama et al. 2007), this man-made action seems unlikely to be the reason. Predators might have crashed the shells open, although there are no observations of bite traces to indicate predation. Thus, the most likely reason for the observed fragmentation is the transportation of dead shells along the river bottom by currents, this process having caused the breakages of especially the shells, which are relative thin at the posterior end. Another aspect of posterior fragmentation is the associated loss of periostracum, evidently shown by a strong positive correlation (Tab. 4). Moreover, the outermost calcareous material may peel off from the rest of the shell, taking with it periostracum. This is supported by a strong correlation between umbonal dissolution and local loss of periostracum (Tab. 4). In this respect, assessments of outer shell surface should be made with caution.

The concepts of actualistic taphonomy (referring to studies on present-day taphonomical processes acting on organisms in contemporary settings to guide the interpretations of their past (e.g. Kowalewski \& LaBarbera 2004) could be useful to understand the occurrence of river mussels during anthropogenic changes to a river basin. Such man-made disturbances are known to have either a direct impact on the river mussels or an indirect one by affecting the populations of fish hosts (see above). At present, the land use at the River Vantaa is mainly forest (59\%) and field (30\%), to lesser extent built-up area $(8 \%)$, water (3\%), and open mire (1\%) (Tikkanen 1989). It is possible that the land use has affected the abundance of river mussels in River Vantaa at least over the last centuries. In such a case, death assemblages could reflect the anticipated anthropogenic changes and as such increase their value as measures for conservation management (Kidwell 2013). In the case of extinct populations, their past occurrences are to be based on death assemblages as accompanied by ${ }^{14} \mathrm{C}$ dating. Our study indicates that death assemblages can be time-averaged without necessarily been drastically reflected in their taphonomical features.

\section{Sampling strategy}

Possibly, there are factors likely to artificially shape the structure of the data set. First, the sampling strategy to originally collect the shells was based on divers' ability to collect the shells from the river bottom where large shells were probably more likely to be hand-picked than very small shells or even the shell fragments that might have been present in the river sediment. This may pose a limitation for the results of study, at least limiting the number of collected young (small) individuals. Second, the shells of $U$. crassus are from nine sampling sites, while those of $U$. tumidus are from the same site. However, the shells did not actually show any notable changes in preservation between the sampling sites. When mussel shells are collected from the river settings, the wetness and acidity of the sites of deposition are known to be 
crucial for favouring the preservation of calcareous and periostracal materials (Nielsen et al. 2008). All the present shells are from the same type of setting, the bottom of the studied river, which is permanently covered by water (Vahtera et al. 2010). This could be the reason why the site-dependent factors did not appear to deviate the shell preservation in this study. Also, the site characteristics (pertaining to $U$. crassus) did not indicate any obvious differences (Helama et al. 2017).

\section{Conclusions}

Death assemblages from the river Vantaanjoki were examined for articulated shells of Unio crassus and U. tumidus. The following main conclusions can be drawn:

(1) The death assemblages comprise relatively large shells, which suggest size selectivity. The reason can be sampling strategy, removal of smaller shells by dissolution, or the absence of small mussels because of insufficient juvenile recruitment.

(2) The taphonomical indices show that the overall preservation is unrelated to the measured ranges of shell size. Site-dependent factors do not appear to influence the shell preservation, indicating no obvious differences.

(3) Differential preservation in individual shells is confirmed. The umbones are obviously affected by corrosion of which area shows a strong correlation between its length and height. The size of the corrosion area is to an extent correlated to the shell size and, therefore, probably to the mussels' ontogenetic age. Also, the posterior end of the shells tends to be more fragmented than other parts, probably related to transportation along the river bottom.

(4) There is no evidence of man-made taphonomical features.

(5) ${ }^{14} \mathrm{C}$ dates of the shells suggest that the phenomenon of time-averaged death assemblages is evident. The actual span of post-mortem ages might be even broader than the pre-modern radiocarbon dates indicate. The possibility of old food effect cannot be exclusively ruled out.

(6) Solely taphonomical features do not provide an obvious way of distinguishing ancient and recent shells in the case of the present samples.

\section{Acknowledgement}

We thank Ilmari Valovirta (Finnish Museum of Natural History, University of Helsinki) for access to the investigated shell material derived via a co-operation with WWF Finland. Support from the Waldemar von Frenckells stiftelse is acknowledged. Eleonor Tietze (Universidad Nacional de Mar del Plata, Argentina) and an anonymous reviewer are thanked for their constructive reviews.

\section{References}

Billett, M.F., Garnett, M.H., Dinsmore, K.J., Dyson, K.E., Harvey, F., Thomson, A.M., Pirrainen, S. \& Kortelainen, P. 2012. Age and source of different forms of carbon released from boreal peatland streams during spring snowmelt in E. Finland. Biogeochemistry 111, 273-286.

DOI 10.1007/s10533-011-9645-4

BJÖRK, S. 1962. Investigations on Margaritifera margaritifera and Unio crassus. Limnologic studies in rivers in South Sweden. Acta Limnologica 4, 1-109.

Briggs, D.J., Gilbertson, D.D. \& Harris, A.L. 1990. Molluscan taphonomy in a braided river environment and its implications for studies of Quaternary cold-stage river deposits. Journal of Biogeography 17(6), 623-637. DOI 10.2307/2845144

Bronk RAmsey, C. 2009. Bayesian analysis of radiocarbon dates. Radiocarbon 51(1), 337-360.

DOI 10.1017/S0033822200033865

Cummins, R.H. 1994. Taphonomic processes in modern freshwater molluscan death assemblages: Implications for the freshwater fossil record. Palaeogeography, Palaeoclimatology, Palaeoecology 108(1-2), 55-73.

DOI 10.1016/0031-0182(94)90022-1

Cuttelod, A., Seddon, M. \& Neubert, E. 2011. European Red List of non-marine molluscs. 97 pp. Publications Office of the European Union, Luxembourg.

Davies, D.J., Powell, E.N. \& Stanton, R.J. JR 1989. Relative rates of shell dissolution and net sediment accumulation a commentary: can shell beds form by the gradual accumulation of biogenic debris on the sea floor? Lethaia 22(2), 207-212. DOI 10.1111/j.1502-3931.1989.tb01683.x

Davis-Foust, S.L., Bruch, R.M., Campana, S.E., Olynyk, R.P. \& JANSSEN, J. 2009. Age validation of freshwater drum using bomb radiocarbon. Transactions of the American Fisheries Society 138(2), 385-396. DOI 10.1577/T08-097.1

Dietl, G.P., Kidwell, S.M., Brenner, M., Burney, D.A., Flessa, K.W., Jackson, S.T. \& Koch, P.L. 2015. Conservation paleobiology: Leveraging knowledge of the past to inform conservation and restoration. Annual Review of Earth and Planetary Sciences 43(1), 79-103.

DOI 10.1146/annurev-earth-040610-133349

Driscoll, E.G. 1970. Selective bivalve destruction in marine environment, a field study. Journal of Sedimentary Petrology 40(3), 898-905. DOI 10.1306/74D720DB-2B21-11D7-8648000102C1865D

ElLIS, A.E. 1978. British Freshwater Bivalve Mollusca. Key and Notes for the Identification of the Species. Synopses of the British Fauna (New series) 11, 1-109. The Linnean Society of London. Academic Press, London.

Erthal, F., Kotzian, C.B. \& Simões, M.G. 2011. Fidelity of 
molluscan assemblages from the Touro Passo Formation (Pleistocene-Holocene), southern Brazil: taphonomy as a tool for discovering natural baselines for freshwater communities. Palaios 26(7), 433-446. DOI 10.2110/palo.2010.p10-145r

Erthal, F., Kotzian, C.B. \& Simões, M.G. 2015. Multi-step taphonomic alterations in fluvial mollusk shells: a case study in the Touro Passo Formation (Pleistocene-Holocene), southern Brazil. Palaios 30(5), 388-402.

DOI 10.2110/palo.2013.104

Flessa, K.W. 2002. Conservation paleobiology. American Paleontologist 10, 2-5.

Flessa, K.W., Cutler, A.H. \& Meldahl, K.H. 1993. Time and taphonomy: quantitative estimates of time-averaging and stratigraphic disorder in a shallow marine habitat. Paleobiology 19(2), 266-286. DOI 10.1017/S0094837300015918

Fürsich, F.T. \& ABERHAN, M. 1990. Significance of time-averaging to palaeocommunity analysis. Lethaia 23(2), 143-152. DOI 10.1111/j.1502-3931.1990.tb01355.x

Fürsich, F.T. \& Kauffman, E.G. 1984. Palaeoecology of marginal marine sedimentary cycles in the Albian Bear River Formation of south-western Wyoming. Palaeontology 27(3), 501-536.

Gomez, A.A., Jaramillo, C.A., Parra, M., \& Mora, A. 2009. Huesser Horizon: a lake and a marine incursion in Northwestern South America during the early Miocene. Palaios 24(3-4), 199-210. DOI 10.2110/palo.2007.p07-074r

Goslar, T. 2010. Report on C-14 dating in the Poznan Radiocarbon Laboratory. Job No. 4672/10. Poznańskie Laboratorium Radiowęglowe, Poznań.

Hallman, D.P., Flessa, K.W., Kowalewski, M., Hertweck, G., Aggen, J. \& CARlton, J. 1996. Ternary taphograms and the comparative taphonomy of recent mollusks from the North Sea and the Gulf of California. Senckenbergiana Maritima $27,67-75$.

Hammer, Ø. 2018. PAST. PAleontological STatistics. Version 3.20. Reference manual. 262 pp. University of Oslo, Oslo. Accessed 16.07.19. https://folk.uio.no/ohammer/past/

Hammer, Ø., Harper, D.A.T. \& Ryan, P.D. 2001. PAST: Paleontological Statistics Software Package for Education and Data Analysis. Palaeontologia Electronica 4(1), 9 pp.

Helama, S. \& Valovirta, I. 2007. Shell morphometry, pre-mortal taphonomy and ontogeny-related growth characteristics of freshwater pearl mussels in northern Finland. Annales Zoologici Fennici 44, 285-302.

Helama, S. \& Valovirta, I. 2014. An autoecological study of annual shell growth increments in Margaritifera margaritifera from Lapland, Subarctic Finland. Memoir of the Fukui Prefectural Dinosaur Museum 13, 25-35.

Helama, S., Nielsen, J.K. \& Valovirta, I. 2007. Conchology of endangered freshwater pearl mussel: Conservational palaeobiology applied to museum shells originating from northern Finland. Bollettino Malacologico 43, 161-170.

Helama, S., Valovirta, I. \& Nielsen, J.K. 2017. Growth characteristics of the endangered thick-shelled river mussel (Unio crassus) near the northern limit of its natural range. Aquatic Conservation: Marine and Freshwater Ecosystems 27(2), 476-491. DOI 10.1002/aqc.2698
Hua, Q., Barbetti, M., \& Rakowski, A.Z. 2013. Atmospheric radiocarbon for the period 1950-2010. Radiocarbon 55(4), 2059-2072. DOI 10.2458/azu_js_rc.v55i2.16177

Hyvärinen, E., Juslén, A., Kemprainen, E., Uddström, A. \& Liukko, U.-M. (eds) 2019. The 2019 Red List of Finnish Species. 704 pp. Ministry of the Environment, Finnish Environment Institute, Helsinki.

KIDwell, S.M. 1998. Time-averaging in the marine fossil record: overview of strategies and uncertainties. Geobios 30(7), 977-995. DOI 10.1016/S0016-6995(97)80219-7

Kidwell, S.M. 2013. Time-averaging and fidelity of modern death assemblages: Building a taphonomic foundation for conservation palaeobiology. Palaeontology 56(3), 487-522. DOI 10.1111/pala.12042

Kidwell, S.M. 2015. Biology in the Anthropocene: Challenges and insights from young fossil records. Proceedings of the National Academy of Sciences of the United States of America 112(16), 4922-4929. DOI 10.1073/pnas.1403660112

Kidwell, S.M. \& Bosence, D.W.J. 1991. Taphonomy and timeaveraging of marine shelly faunas: 115-209. In Allison, P.A. \& Briggs, D.E.G. (eds) Taphonomy: Releasing the data locked in the fossil record. Plenum Press, New York. DOI 10.1007/978-1-4899-5034-5_4

Knudsen, J., Jensen, K.R., Nielsen, C. \& Johnson, R.I. 2003. Lorentz Spengler's descriptions of freshwater mussels (Mollusca: Unionacea): translation and notes. Steenstrupia 27(2), 263-279.

Kotzian, C.B. \& Simões, M.G. 2006. Taphonomy of recent freshwater molluscan death assemblages, Touro Passo Stream, southern Brazil. Revista Brasileira de Paleontologia 9(2), 243-260. DOI 10.4072/rbp.2006.2.08

Kowalewski, M. \& LaBarbera, M. 2004. Actualistic taphonomy: death, decay, and disintegration in contemporary settings. Palaios 19(5), 423-427. DOI 10.1669/0883-1351(2004)019<0423:ATDDAD>2.0.CO;2

Kowalewski, M., Flessa, K.W. \& Hallman, D.P. 1995. Ternary taphograms: triangular diagrams applied to taphonomic analysis. Palaios 10(5), 478-483. DOI 10.2307/3515049

Kusnerik, K.M., Means, G.H, Portell, R.W., Brenner, M., Hua, Q., Kannai, A., Means, R., Monroe, M.A., KowalewSKI, M. 2020. Live, dead, and fossil mollusks in Florida freshwater springs and spring-fed rivers: Taphonomic pathways and the formation of multisourced, time-averaged death assemblages. Paleobiology 2020, 1-23.

DOI 10.1017/pab.2020.25

LARsen, T., Yokoyama, Y \& Fernandes, R. 2018. Radiocarbon in ecology: Insights and perspectives from aquatic and terrestrial studies. Methods in Ecology and Evolution 9, 181-190. DOI 10.1111/2041-210X.12851

Linnaeus, C. 1758. Systema naturce per regna tria naturce, secundum classes, ordines, genera, species, cum characteribus, differentiis, synonymis, locis. $10^{\text {th }}$ edition, $1,824 \mathrm{pp}$. Laurentii Salvii, Holmiæ. DOI 10.5962/bhl.title.542

Lopes-Lima, M., Kebapçi, U. \& Van Damme, D. 2014. Unio crassus. The IUCN Red List of Threatened Species 2014, e.T22736A42465628.

DOI 10.2305/IUCN.UK.2014-1.RLTS.T22736A42465628.en 
Lopes-Lima, M., Sousa, R., Geist, J., Aldridge, D.C., Araujo, R., Bergengren, J., Bespalaya, Y., Bódis, E., Burlakova, L., Van Damme, D., Douda, K., Froufe, E., Georgiev, D., Gumpinger, C., Karatayev, A., Kebapçi, Ü., Killeen, I., Lajtner, J., Larsen, B.M., Lauceri, R., Legakis, A., Lois, S., LundberG, S., Moorkens, E., Motte, G., Nagel, K.-O., Ondina, P., Outeiro, A., Paunovic, M., Prié, V., von Proschwitz, T., Riccardi, N., Rudzīte, M., Rudzītis, M., Scheder, C., Seddon, M., Şereflişan, H., Simić, V., Sokolova, S., Stoeckl, K., Taskinen, J., Teixeira, A., Thielen, F., Trichkova, T., Varandas, S., Vicentini, H., Zajac, K., Zajac, T. \& Zogaris, S. 2017. Conservation status of freshwater mussels in Europe: state of the art and future challenges. Biological Reviews 92(1), 572-607. DOI 10.1111/brv.12244

Mandahl-Barth, G. 1949. Muslinger (Lamellibranchia, Bivalvia, Acephala), 114-175. In Mandahl-Barth, G. \& BondeSEN, P. (eds) Bløddyr III Ferskvandsbløddyr. Danmarks Fauna 54.

Martello, A.R., Kotzian, C.B. \& Simões, M.G. 2006. Quantitative fidelity of Recent freshwater mollusk assemblages from the Touro Passo River, Rio Grande do Sul, Brazil. Iheringia, Série Zoologia 96(4), 453-465. DOI 10.1590/S0073-47212006000400010

Nielsen, J.K. \& Funder, S. 2003. Taphonomy of Eemian marine molluscs and acorn barnacles from eastern Arkhangelsk region, northern Russia. Palaeogeography, Palaeoclimatology, Palaeoecology 191(2), 139-168.

DOI 10.1016/S0031-0182(02)00707-1

Nielsen, J.K., Helama, S. \& Nielsen, J.K. 2008. Taphonomy of freshwater molluscs in carbonate-poor deposits: a case study of the river pearl mussel in northeastern Finnish Lapland. Norwegian Journal of Geology 88(2), 103-116.

Nielsen, J.K., Nielsen, J.K., Evison, K., Hanken, N.-M. \& HeLAMA, S. 2018. An invertebrate macrofauna from an early Holocene fjord-delta system, North Norway. Bulletin of Geosciences 93(1), 27-52. DOI 10.3140/bull.geosci.1677

Nordenskiöld, A.E. \& Nylander, A.E. 1856. Finlands Mollusker. 116 pp. J. Simelii Arfvingar, Helsingfors.

Olszewski, T. 1999. Taking advantage of time-averaging. Paleobiology 25(2), 226-238.

DOI $10.1017 / \mathrm{S} 009483730002652 \mathrm{X}$

OlszewsKi, T.D. 2004. Modeling the influence of taphonomic destruction, reworking, and burial on time-averaging in fossil accumulations. Palaios 19(1), 39-50.

DOI 10.1669/0883-1351(2004)019<0039:MTIOTD>2.0.CO;2

PekKarinen, M. 1991. Annual ring formation in some unionacean shells in the River Vantaanjoki (southern Finland). Bivalve Studies in Finland 1, 10-19.

Pekrarinen, M. 1993. Reproduction and condition of unionid mussels in the Vantaa River, South Finland. Archiv für Hydrobiologie 127(3), 357-375.

Philipsson, L.M. 1788. Dissertatio historico-naturalis sistens nova testaceorum genera. Quam venia ampliss. facult. philosophica praside D.M. Andr. J. Retzio. Ad publicum examen defert Laurentius Münter Philipsson. 23 pp. Berlingianis, Lundæ.

PIP, E. 1988. Differential attrition of molluscan shells in fresh- water sediments. Canadian Journal of Earth Sciences 25(1), 68-73. DOI 10.1139/e88-007

Press, W.H., Teukolsky, S.A., Vetterling, W.T. \& Flannery, B.P. 1992. Numerical recipes in C. The art of scientific computing. 994 pp. Cambridge, University Press, $2^{\text {nd }}$ Edition.

RAFINESQUE, C.S. 1820. Monographie des coquilles bivalves fluviatiles de la rivière Ohio, contenant douze genres et soixanteneuf espèces [Title correction p. 417]. Annales Générales des Sciences Physiques, De l'Imprimerie de Weissenbruch, Père, Bruxelles 5, 287-322.

RAMSEY, P.H. 1989. Critical values for Spearman's rank order correlation. Journal of Educational Statistics 14(3), 245-253. DOI 10.3102/10769986014003245

Reimer, P.J., Brown, T.A. \& Reimer, R.W. 2004. Discussion: Reporting and calibration of post-bomb ${ }^{14} \mathrm{C}$ data. Radiocarbon 46(3), 1299-1304. DOI 10.1017/S0033822200033154

Reimer, P.J., Bard, E., Bayliss, A., Beck, J.W., Blackwell, P.G., Bronk Ramsey, C., Buck, C.E., Cheng, H., Edwards, R.L., Friedrich, M., Grootes, P.M., Guilderson, T.P., Haflidason, H., Hajdas, I., Hatté, C., Heaton, T.J., Hoffmann, D.L., Hogg, A.G., Hughen, K.A., Kaiser, K.F., Kromer, B., Manning, S.W., Niu, M., Reimer, R.W., Richards, D.A., Scott, E.M., Southon, J.R., Staff, R.A., Turney, C.S.M., \& van der Plicht, J. 2013. IntCal13 and Marine13 radiocarbon age calibration curves 0-50,000 years cal BP. Radiocarbon 55(4), 1869-1887. DOI 10.2458/azu_js_rc.55.16947

Stenström, K.E., Skog, G., Georgiadou, E., Genberg, J., \& JoHANSSON, A. 2011. A guide to radiocarbon units and calculations. 17 pp. Department of Physics, Division of Nuclear Physics, Lund University, internal report LUNFD6, NFFR-3111.

SzABÓ, K. 2017. Molluscan shells as raw materials for artefact production, 308-325. In AlLen, M.J. (ed.) Molluscs in Archaeology. Methods, approaches and applications. Oxbow Books, Oxford. Studying Scientific Archaeology 3. DOI $10.2307 /$ j.ctvh $1 \mathrm{dk} 5 \mathrm{~s} .24$

Tietze, E. \& De Francesco, C.G. 2012. Compositional fidelity of subfossil mollusk assemblages in streams and lakes of the southeastern Pampas, Argentina. Palaios 27, 401-413. DOI 10.2110/palo.2011.p11-124r

TikKanEn, M. 1989. Geomorphology of the Vantaanjoki drainage basin, southern Finland. Fennia 167(1), 19-72.

TikKanen, M. \& Ruth, O. 2003. Origins and development of the ancient outflow channel of the river Vantaanjoki, southern Finland, as indicated by fluvial sediments. Fennia 181(1), 69-83.

Vahtera, H., Männynsalo, J. \& Lahti, K. 2010. Vantaanjoen yhteistarkkailu Vedenlaatu vuosina 2005-2009. Vantaanjoen ja Helsingin seudun vesiensuojeluyhdistys ry 64, 126 pp. Accessed 16.07.19. http://www.vhvsy.fi/files/upload_pdf/1621/ vy2009.pdf

Valovirta, I. 2008. Vantaanjoen Natura-alueen vuollejokisimpukkainventointi 2004-2007. 48 pp. Finnish Museum of Natural History, University of Helsinki.

VAn Damme, D. 2011. Unio tumidus. The IUCN Red List of Threatened Species 2011, e.T156111A4898810.

DOI 10.2305/IUCN.UK.2011-2.RLTS.T156111A4898810.en 
Welter Schultes, F. 2013. Species summary for Unio crassus. AnimalBase. Accessed 16.07.19. http://www.animalbase. uni-goettingen.de/zooweb/servlet/AnimalBase/home/ species? $\mathrm{id}=1561$

Wesenberg-Lund, C. 1937. Ferskvandsfaunaen biologisk belyst. Invertebrata. 837 pp. 2 vols, Nordisk Forlag, København.

Wesselingh, F.P., KaAndorp, R.J.G., Vonhof, H.B., Räsänen, M.E., Renema, W. \& Gingras, M. 2006. The nature of aquatic landscapes in the Miocene of western Amazonia: an integrated palaeontological and geochemical approach. Scripta Geologica 133, 363-393.

Westerlund, C.A. 1871-1873. Fauna Molluscorum Terrestrium et Fluviatilium Svecice, Norvegice et Danice. Sveriges, Norges och Danmarks Land- och Sötvatten-mollusker. I. Landmolluskerna. II. Sötvattenmollusker. 651 pp. Berlingska Boktryckeriet, Lund. DOI 10.5962/bhl.title.13242

WilLis, K.J. \& BIRKs, H.J.B. 2006. What is natural? The need for a long-term perspective in biodiversity conservation. Science 314, 1261-1265. DOI 10.1126/science.1122667

Wolverton, S., Randklev, C.R. \& Kennedy, J.H. 2010. A conceptual model for freshwater mussel (family: Unionidae) remain preservation in zooarchaeological assemblages. Journal of Archaeological Science 37, 164-173.

DOI 10.1016/j.jas.2009.09.028 